Pure and Applied Mathematics Quarterly

Volume 4, Number 2

(Special Issue: In honor of

Fedor Bogomolov, Part 1 of 2)

$383-425,2008$

\title{
The Regeneration of a 5-point
}

\author{
Michael Friedman and Mina Teicher ${ }^{1}$ \\ Dedicated to Fedor Bogomolov on the occasion of his 60th birthday
}

\begin{abstract}
The braid monodromy factorization of the branch curve of a surface of general type is known to be an invariant that completely determines the diffeomorphism type of the surface (see [2]). Calculating this factorization is of high technical complexity; computing the braid monodromy factorization of branch curves of surfaces uncovers new facts and invariants of the surfaces. Since finding the branch curve of a surface is very difficult, we degenerate the surface into a union of planes. Thus, we can find the braid monodromy of the branch curve of the degenerated surface, which is a union of lines. The regeneration of the singularities of the branch curve, studied locally, leads us to find the global braid monodromy factorization of the branch curve of the original surface. So far, only the regeneration of the BMF of 3,4 and 6-point (a singular point which is the intersection of 3 / 4 / 6 planes; see [6],[8]) were done. In this paper, we fill the gap and find the braid monodromy of the regeneration of a 5-point. This is of great importance to the understanding of the BMT (braid monodromy type) of surfaces [2].

This braid monodromy will be used to find the global braid monodromy factorization of different surfaces; in particular - the monodromy of the branch curve of the Hirzebruch surface $F_{2,(2,2)}$.
\end{abstract}

Received March 8, 2006.

${ }^{1}$ This work was supported Supported by the Emmy Noether Institute Fellowship (by the Minerva Foundation of Germany) and the Israel Science Foundation grant \#8008/02-3 (Excellency Center Group Theoretic Methods in the Study of Algebraic Varieties). 


\section{IntroduCtion}

Let $X \subset \mathbb{C P}^{N}$ be a smooth algebraic surface of degree $n$. One may obtain information on $X$ by considering it as a branched cover of another surface. If the base surface is $\mathbb{C P}^{2}$ and if the map $X \rightarrow \mathbb{C P}^{2}$ is a generic projection, then the branch locus is a plane curve $\bar{S} \subset \mathbb{C P}^{2}$ which is, in general, singular. If the projection is generic, the singularities are nodes and cusps. Let $S \subset \mathbb{C}^{2} \subset \mathbb{C P}^{2}$ denote a generic affine portion of $\bar{S}$. A general problem is the study of the fundamental groups of the complement of the branch curve: $\pi_{1}\left(\mathbb{C}^{2}-S\right)$ and $\pi_{1}\left(\mathbb{C P}^{2}-\bar{S}\right)$.

It has been proven that these fundamental groups (derived from braid monodromy factorizations) are invariants that distinguish between diffeomorphic surfaces (see [2]); that is, if two surfaces have equivalent braid monodromy factorizations (and thus isomorphic fundamental groups), then they are diffeomorphic. However, the converse is not true, that is, the diffeomorphism type does not determine the equivalence class of the factorization. In [3] a pair of diffeomorphic surfaces was constructed such that the braid monodromy factorizations are not equivalent.

The above fundamental groups cannot be found directly, since finding the branch curve explicitely is very difficult. Therefore, one has to degenerate the surface $X$ into a union of planes, where in this case, the branch curve is easy to find - it is an arrangment of lines. It is known, by the Zariski-Van Kampen Theorem that the braid monodromy factorization (BMF ; see Section 2 for its definition) of the branch curve determines the desired fundamental groups. Note that the BMF of any curve is given by a product of the local BMF in the neighborhood of the singular points of the branch curve. Thus, the BMF of any line arrangement can be found explicitly (see [5]). By applying the regeneration techniques on the singularities of the arrangement of lines, one can find the BMF of the original branch curve.

So it is very important to find out what are the local BMF that are obtained from regenerating different line arrangements (or line and conic arrangement).

Till now, most of the arrangements that include one line and one conic (or two lines) were treated. In [6] the BMFs of the regeneration of a tangent / node / branch point are given. However, for more complicated arrangements, only a 
few results are known. The BMF of the regeneration of a standard 3-point (that is, a singular point which is the intersection of 3 planes), 4-point and 6-point are presented in [8]. Figure I.1 is a depiction of the arrangement of planes (that correspond to the regions delimited by the edges of the diagram) and edges (that correspond to lines of intersection between two planes).

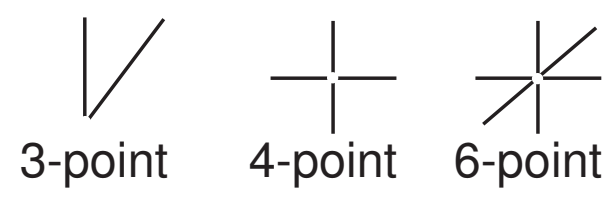

(figure I.1)

Notice that in the cases in which the line arrangements include more than two lines, the order of the regeneration effects how the factorization will look.

In this article we compute two important braid monodromy factorizations, which were not known till now - the BMF of a 5-point, and a general formula of a certain type of $(k+1)$-point where $k \geq 2$.

This article is organized as follows: In Section 2 we give the main definitions (BMF and regeneration techniques), and then we compute the BMF of the two main cases that were mentioned above. Section 3 shows the importance of these factorizations by introducing an example which uses one of them.

Acknowledgment: The authors wish to thank Prof. Eugenii Shustin for his help and for fruitful discussions.

\section{5-POINT REGENERATION}

This section introduces the main result of the article - the local braid monodromy factorization induced from the regeneration of a neighborhood of a 5point; that is, a point which is the intersection of 5 planes. Our result deals with two specific cases for which this situation can appear, though there are other configurations of 5-planes passing through a point. Note that we actually consider this point to be a singular point of the branch curve of a degenerated surface, when considering its generic projection to $\mathbb{C P}^{2}$. But first we need to recall a few definitions, related to the braid monodromy factorization and to the regeneration techniques. 
2.1. Preliminaries: $\mathbf{B M F}$ and regeneration techniques. Computing the braid monodromy is the main tool to compute fundamental groups of complements of curves. In this subsection we define the braid monodromy.

Let $D$ be a closed disk in $\mathbb{R}^{2}, K \subset \operatorname{Int}(D), K$ finite, $n=\# K$. Recall that the braid group $B_{n}[D, K]$ can be defined as the group of all equivalent diffeomorphisms $\beta$ of $D$ such that $\beta(K)=K,\left.\beta\right|_{\partial D}=\left.\operatorname{Id}\right|_{\partial D}$.

Definition: $\underline{H(\sigma), \text { half-twist defined by } \sigma}$

Let $a, b \in K$, and let $\sigma$ be a smooth simple path in $\operatorname{Int}(D)$ connecting $a$ with $b$ s.t. $\sigma \cap K=\{a, b\}$. Choose a small regular neighborhood $U$ of $\sigma$ contained in $\operatorname{Int}(D)$, s.t. $U \cap K=\{a, b\}$. Denote by $H(\sigma)$ the diffeomorphism of $D$ which switches $a$ and $b$ by a counterclockwise 180 degree rotation and is the identity on $D \backslash U$. Thus it defines an element of $B_{n}[D, K]$, called the half-twist defined by $\sigma$

Assume that all of the points of $K$ are on the $X$-axis (when considering $D$ in $\mathbb{R}^{2}$ ). In this situation, if $a, b \in K$, and $z_{a, b}$ is a path that connects them, then we denote it by $Z_{a, b}=H\left(z_{a, b}\right)$. If $z_{a, b}$ is a path that goes below the $x$-axis, then we denote it by $\underline{Z}_{a, b}$, or just $Z_{a, b}$. If $z_{a, b}$ is a path that goes above the $x$-axis, then we denote it by $\bar{Z}_{a, b}$. See [6], Section 2 for additional notations.

Definition: The braid monodromy w.r.t. $S, \pi, u$

Let $S$ be a curve, $S \subseteq \mathbb{C}^{2}$ Let $\pi: S \rightarrow \mathbb{C}^{1}$ be defined by $\pi(x, y)=x$. We denote $\operatorname{deg} \pi$ by $m$. Let $N=\left\{x \in \mathbb{C}^{1} \mid \# \pi^{-1}(x)<m\right\}$. Take $u \notin N$, s.t. $\Re(x) \ll u$ $\forall x \in N$. Let $\mathbb{C}_{u}^{1}=\{(u, y)\}$. There is a natural defined homomorphism

$$
\pi_{1}\left(\mathbb{C}^{1}-N, u\right) \stackrel{\varphi}{\rightarrow} B_{m}\left[\mathbb{C}_{u}^{1}, \mathbb{C}_{u}^{1} \cap S\right]
$$

which is called the braid monodromy w.r.t. $S, \pi, u$, where $B_{m}$ is the braid group. We sometimes denote $\varphi$ by $\varphi_{u}$. In fact, denoting by $E-$ a big disk in $\mathbb{C}^{1}$ s.t. $E \supset N$, we can also take the path in $E \backslash N$ not to be a loop, but just a non-selfintersecting path; this induces a diffeomorphism between the models $(D, K)$ at the two ends of the considered path, where $D$ is a big disk in $\mathbb{C}_{u}^{1}$, and $K=\mathbb{C}_{u}^{1} \cap S \subset D$. 
Definition: $\psi_{T}$, Lefschetz diffeomorphism induced by a path $T$

Let $T$ be a path in $E \backslash N$ connecting $x_{0}$ with $x_{1}, T:[0,1] \rightarrow E \backslash N$. There exists a continuous family of diffeomorphisms $\psi_{(t)}: D \rightarrow D, t \in[0,1]$, such that $\psi_{(0)}=I d, \psi_{(t)}\left(K\left(x_{0}\right)\right)=K(T(t))$ for all $t \in[0,1]$, and $\psi_{(t)}(y)=y$ for all $y \in \pi_{1} D$. For emphasis we write $\psi_{(t)}:\left(D, K\left(x_{0}\right)\right) \rightarrow(D, K(T(t))$. Lefschetz diffeomorphism induced by a path $T$ is the diffeomorphism

$$
\psi_{T}=\psi_{(1)}:\left(D, K\left(x_{0}\right)\right) \underset{\sim}{\longrightarrow}\left(D, K\left(x_{1}\right)\right) .
$$

Since $\psi_{(t)}\left(K\left(x_{0}\right)\right)=K(T(t))$ for all $t \in[0,1]$, we have a family of canonical isomorphisms

$$
\psi_{(t)}^{\nu}: B_{p}\left[D, K\left(x_{0}\right)\right] \underset{\sim}{\longrightarrow} B_{p}[D, K(T(t))], \quad \text { for all } t \in[0,1] .
$$

see the following figure for illustration of the above definitions:
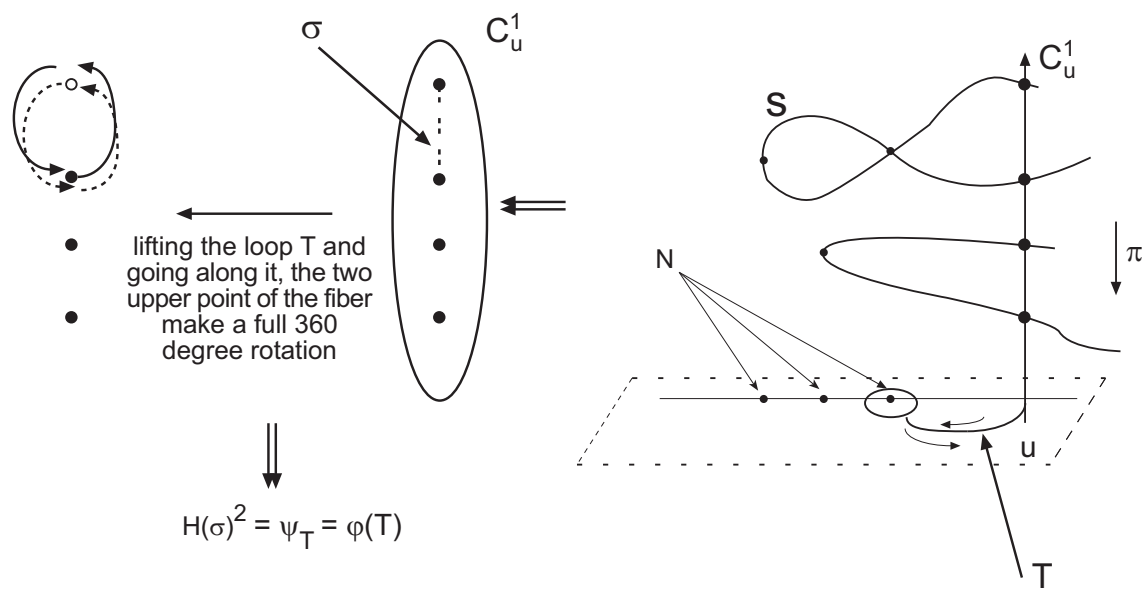

We recall Artin's theorem on the presentation of the Dehn twist of the braid group as a product of braid monodromy elements of a geometric-base (a base of $\pi_{1}=\pi_{1}\left(\mathbb{C}^{1}-N, u\right)$ with certain properties; see [5] for definitions).

Theorem: Let $S$ be a curve transversal to the line in infinity, and $\varphi$ is a braid monodromy of $S, \varphi: \pi_{1} \rightarrow B_{m}$. Let $\delta_{i}$ be a geometric (free) base (g-base) of $\pi_{1}$, and $\Delta^{2}$ is the generator of $\operatorname{Center}\left(B_{m}\right)$. Then:

$$
\Delta^{2}=\prod \varphi\left(\delta_{i}\right) .
$$


This product is also defined as the braid monodromy factorization (BMF) related to a curve $S$.

So in order to find out what is the braid monodromy factorization of $\Delta_{p}^{2}$, we have to find out what are $\varphi\left(\delta_{i}\right), \forall i$. We refer the reader to the definition of a skeleton (see [6]) $\lambda_{x_{j}}, x_{j} \in N$, which is a model of a set of paths connecting points in the fiber, s.t. all those points coincide when approaching $A_{j}=\left(x_{j}, y_{j}\right) \in S$, when we approach this point from the right. To describe this situation in greater detail, for $x_{j} \in N$, let $x_{j}^{\prime}=x_{j}+\alpha$. So the skeleton in $x_{j}$ is defined as system of paths connecting the points in $K\left(x_{j}^{\prime}\right) \cap D\left(A_{j}, \varepsilon\right)$ when $0<\alpha \ll \varepsilon \ll 1, D\left(A_{j}, \varepsilon\right)$ is a disk centered in $A_{j}$ with radius $\varepsilon$.

For a given skeleton, we denote by $\Delta\left\langle\lambda_{x_{j}}\right\rangle$ the braid by rotates by 180 degrees counterclockwise a small neighborhood of the given skeleton. Note that of $\lambda_{x_{j}}$ is a single path, then $\Delta\left\langle\lambda_{x_{j}}\right\rangle=H\left(\lambda_{x_{j}}\right)$.

We also refer the reader to the definition of $\delta_{x_{0}}$, for $x_{0} \in N$ (see [6]), which describes the Lefschetz diffeomorphism induced by a path going below $x_{0}$, for different types of singular points (tangent, node, branch; for example, when going below a node, a half-twist of the skeleton occurs. When going below a tangent point, a full-twist occurs).

We define, for $x_{0} \in N$, the following number: $\varepsilon_{x_{0}}=1,2,4$ when $\left(x_{0}, y_{0}\right)$ is a branch / node / tangent point (respectively). So we have the following statement (see [6], prop. 1.5):

Let $\gamma_{j}$ be a path below the real line from $x_{j}$ to $u$, s.t. $\ell\left(\gamma_{j}\right)=\delta_{j}$. So -

$$
\varphi_{u}\left(\delta_{j}\right)=\varphi\left(\delta_{j}\right)=\Delta<\left(\lambda_{x_{j}}\right)\left(\prod_{m=j-1}^{1} \delta_{x_{m}}\right)>^{\varepsilon_{x_{j}}} .
$$

When denoting $\xi_{x_{j}}=\left(\lambda_{x_{j}}\right)\left(\prod_{m=j-1}^{1} \delta_{x_{m}}\right)$ we get -

$$
\varphi\left(\delta_{j}\right)=\Delta\left\langle\left(\xi_{x_{j}}\right)\right\rangle^{\varepsilon_{x_{j}}} .
$$

Note that the last formula gives an algorithm to compute the wanted factorization. 
For a detailed explanation of the braid monodromy, see [5].

We recall now the regeneration methods.

The regeneration methods are actually, locally, the reverse process of the degeneration method. When regenerating a singular configuration consisting of lines and conics, the final stage in the regeneration process involves doubling each line, so that each point of $K$ corresponding to a line labelled $i$ is replaced by a pair of points, labelled $i$ and $i^{\prime}$. The purpose of the regeneration rules is to explain how the braid monodromy behaves when lines are doubled in this manner. We denote by $Z_{i, j}=H\left(z_{i, j}\right)$ where $z_{i, j}$ is a path connecting points in $K$.

The rules are (see [8], pg. 336-7):

(1) First regeneration rule: The regeneration of a branch point of hyperbola:

A factor of the braid monodromy of the form $Z_{i j}$ is replaced in the regeneration by $Z_{i^{\prime} j} \cdot \stackrel{(j)}{Z}_{i j^{\prime}}$

(2) Second regeneration rule:The regeneration of a node:

A factor of the form $Z_{i j}^{2}$ is replaced by a factorized expression $Z_{i i^{\prime}, j}^{2}:=$ $Z_{i^{\prime} j}^{2} \cdot Z_{i j}^{2}, Z_{i, j j^{\prime}}^{2}:=Z_{i j^{\prime}}^{2} \cdot Z_{i j}^{2}$ or by $Z_{i i^{\prime}, j j^{\prime}}^{2}:=Z_{i^{\prime} j^{\prime}}^{2} \cdot Z_{i j^{\prime}}^{2} Z_{i^{\prime} j}^{2} \cdot Z_{i j}^{2}$.

(3) Third regeneration rule:The regeneration of a tangent point:

A factor of the form $Z_{i j}^{4}$ in the braid monodromy factorized expression is replaced by $Z_{i, j j^{\prime}}^{3}:=\left(Z_{i j}^{3}\right)^{Z_{j j^{\prime}}} \cdot\left(Z_{i j}^{3}\right) \cdot\left(Z_{i j}^{3}\right)^{Z_{j j^{\prime}}}$.

As a result, we get a factorized expression, which, by [2], determines the diffeomorphism type of our surface, and, by [13], determines $\pi_{1}\left(\mathbb{C P}^{2}-\bar{S}\right)$.

2.2. The first case. In this subsection we will look at the case where, locally, we have 5 planes corresponding to the angular sectors of the figure, intersecting each other along a line whenever they have a common edge. The lines $L_{i}, 1 \leq i \leq 5$ are numerated as following: 


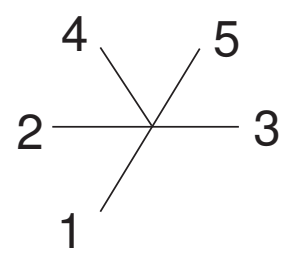

(figure 1)

The lines are numerated in a way such that it describes the respective positions of the points where they intersect $\mathbb{C}_{u}^{1}$. We know that a 5 -point of this sort can be generated during the process of a degeneration of a surface into a union of planes. Thus, when we examine the local braid monodromy factorization of this 5-point, before degenerating, we know it is $\Delta^{2}\langle 1,5\rangle$. So by knowing what the regeneration process will do to this factorization, we will know part of the relations which are in the local fundamental group of $\mathbb{C}^{2}$ (or $\mathbb{C P}^{2}$ ) minus the branch curve in this local neighborhood. Note that in the regeneration process, line 4 is regenerated first, then lines 2 and 3 and then lines 1 and 5 .

In order to compute the desired factorization, we need a few corollaries. The first is cited from [8], and deals with the result of the regeneration process under certain conditions.

Corollary 2.1. Let $V$ be a projective algebraic surface, $D^{\prime}-$ a curve in $V$. Let $f: V \rightarrow \mathbb{C P}^{2}$ be a generic projection. Let $S \subseteq \mathbb{C P}^{2}, S^{\prime} \subseteq V$ be the corresponding branch / ramification curve of $f$. Assume $S^{\prime}$ intersects $D^{\prime}$ in $\alpha^{\prime}$. Let $D=$ $f\left(D^{\prime}\right), \alpha=f\left(\alpha^{\prime}\right)$. Assume that there exist neighborhoods of $\alpha$ and $\alpha^{\prime}$ s.t. $\left.f\right|_{S^{\prime}}$ and $\left.f\right|_{D^{\prime}}$ are isomorphic. Then $D$ is tangent to $S$ at $\alpha$.

Proof: see [8].

The second corollary deals with the computation of a few braids, which are induced from loops going around a complex intersection of a conic and a line. We need this lemaa, since this situation appears during the regeneration process. So consider the following model.

Let $C=\left\{\left(y^{2}-x\right)(y+x+1)=0\right\}, \pi_{1}, \pi_{2}: C \rightarrow \mathbb{C}, \pi_{1}(x, y) \mapsto x, \pi_{2}(x, y) \mapsto y$. Denote by $p_{1}, p_{2}$ the points of intersection of $y^{2}=x$ and $y=-x-1$. So $x_{p_{1}}=-\frac{1}{2}+\frac{\sqrt{3} i}{2}, x_{p_{2}}=-\frac{1}{2}-\frac{\sqrt{3} i}{2}$. Denote $-x_{0}=-\frac{1}{4}, A=\pi_{2}\left(\pi_{1}^{-1}\left(x_{0}\right)\right)=$ $\left\{ \pm \frac{1}{2} i,-\frac{3}{4}\right\}, x_{1}=-\frac{3}{4}, A^{\prime}=\pi_{2}\left(\pi_{1}^{-1}\left(x_{1}\right)\right)=\left\{ \pm \frac{\sqrt{3}}{2} i,-\frac{1}{4}\right\}$ (see figure 2 ). 


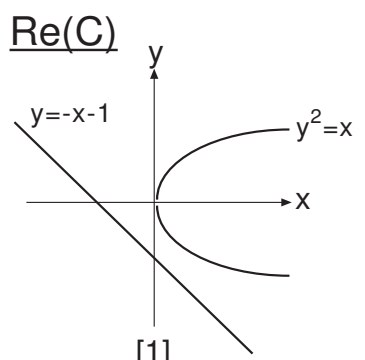

[1]

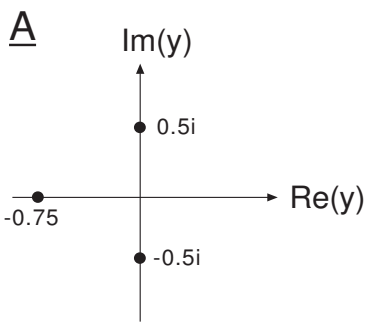

[2]

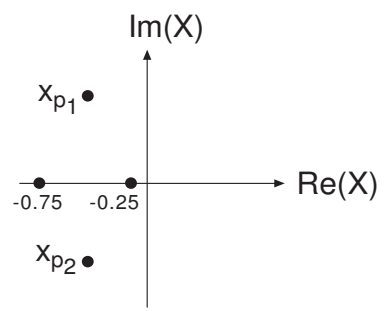

[3]

(figure 2)

Remark: Note that $A^{\prime}$ and $A$ (which are on the $Y$-axis) are equivalent in the sense that if $a \in A, a^{\prime} \in A^{\prime}$ and $\Re(a), \Re\left(a^{\prime}\right) \neq 0$ (or $\Im(a), \Im\left(a^{\prime}\right)>0$ or $\left.\Im(a), \Im\left(a^{\prime}\right)<0\right)$ ), then $a$ and $a^{\prime}$ come from the same component of $C$. Let $D$ be a disk on the Y-axis s.t. $A, A^{\prime} \subset D$. Thus we can define a continuous diffeomorphism $\beta: D \rightarrow D$ s.t. $\beta\left(\frac{-3}{4}\right)=\frac{-1}{4}, \beta\left(\frac{ \pm i}{2}\right)=\frac{ \pm \sqrt{3} i}{2}, \beta(\partial D)=\partial D, \beta(\Im(Y) \cap D)=\Im(Y) \cap D, \beta(\Re(Y) \cap$ $D)=\Re(Y) \cap D$, and outside a small neighborhood of the $\Im(Y)-$ and $\Re(Y)$-axis, $\beta=I d$.

Denote:

$\sigma_{1}$ - the segment connecting $-\frac{3}{4}$ and $\frac{i}{2}$ in $A$;

$\sigma_{2}$ - the segment connecting $-\frac{3}{4}$ and $\frac{-i}{2}$ in $A$; See figure 3.[1]

$\sigma_{1}^{\prime}$ - the segment connecting $-\frac{1}{4}$ and $\frac{\sqrt{3} i}{2}$ in $A^{\prime}$;

$\sigma_{2}^{\prime}$ - the segment connecting $-\frac{1}{4}$ and $-\frac{\sqrt{3} i}{2}$ in $A^{\prime}$.

Let $l_{1}(t), l_{2}(t),(0 \leq t \leq 1)$ be two loops starting (and ending) at $x_{0}$, s.t. $l_{i}$ is around $x_{p_{i}}$. Lifting $l_{i}(i=1,2)$ to $C$ and projecting it to $\mathrm{D}$, we get a motion: $(D, A) \rightarrow(D, A)$, which induces a braid; let $l_{3}(t),(0 \leq t \leq 1)$ be a curve starting at $x_{1}$, ending at $x_{0}$ and surrounding $x_{p_{2}}$ from below (see figures 3.[2], 3. [3]).

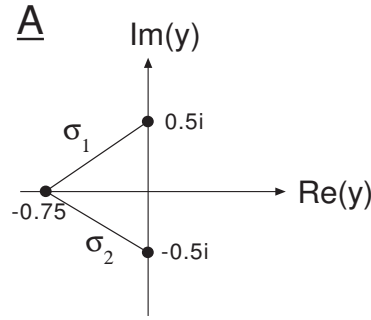

[1]

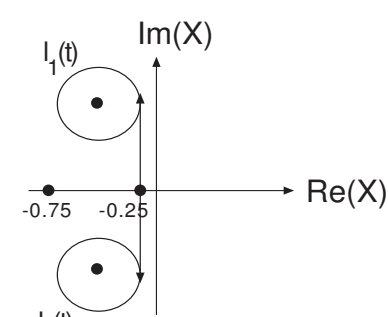

[2]

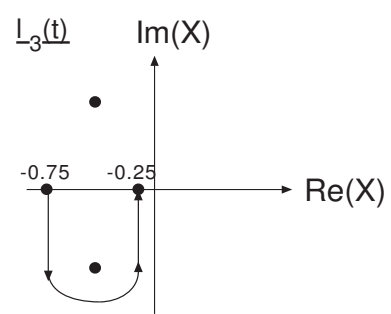

[3]

(figure 3) 
As above, we get a motion $(D, A) \rightarrow\left(D, A^{\prime}\right)$. Denote:

$\psi_{l_{1}}, \psi_{l_{2}}$ - the motions induced from $l_{1}, l_{2}$ (that is, the Lefschetz isomorphisms induced by the paths; we omit the superscript, as mentioned in the introduction). $\psi_{l_{3}}$ - the motion induced from $l_{3}$ (after composing $\beta$ on the resulting disk).

Corollary 2.2. $\psi_{l_{1}}=H\left(\sigma_{2}\right)^{2}, \psi_{l_{2}}=H\left(\sigma_{1}\right)^{2}, \psi_{l_{3}}=H\left(\sigma_{1}^{\prime}\right)^{2}$, where $H(\sigma)$ is the halftwist induced from the path $\sigma$.

Proof: For $\psi_{l_{1}}, \psi_{l_{2}}$ we can look at a small neighborhood of $p_{1}\left(p_{2}\right)$. Since in this small neighborhood the intersection of the branch of the conic, which $p_{1}\left(p_{2}\right)$ lies on, and the line can be treated as the intersection of 2 lines, it is obvious that when we perform a full loop around $x_{p_{1}}$ or around $x_{p_{2}}$, the induced motion of the above points (points $0.5 i$ and -0.75 or points $-0.5 i$ and -0.75 ) is a rotation of 360 degrees, and the induced braid is $H(\sigma)^{2}$ where $\sigma$ is the path connecting the points $\left(\sigma_{2}\right.$ or $\sigma_{1}$; see figure 3.[1]). Indeed, the line is $y=-x-1$, so when $x$ moves along $l_{1}$, the corresponding value of $y$ is in the lower half-plane; both end-points of $\sigma_{2}$ approach $-\frac{1}{2}-\frac{\sqrt{3}}{2} i$ as $x$ approaches $x_{p_{1}}$; And similarly for $l_{2}$.

We shall now compute $\psi_{l_{3}}$. Observe that the union of the straight line from $x_{0}$ to $x_{1}$ with the arc $l_{3}$ is a closed loop from $x_{0}$ to itself, homotopic to $l_{2}$. Moving $x$ along the real axis from $x_{0}$ to $x_{1}$ induces the diffeomorphism $\beta:(D, A) \rightarrow\left(D, A^{\prime}\right)$ introduced earlier, so (up to isotopy) $\psi_{l_{3}} \circ \beta=\psi_{l_{2}}$, which gives $\psi_{l_{3}}$.

So we now look at a point $v$, which is the intersection of 5 lines (see figure 1), which are (part of a) branch curve of a degenerated surface. Since we consider that this branch curve is a result of a degeneration process, we can apply Corollary 2.1 when we are trying to find out what will happen when we first regenerate line 4 .

Denote $V=\bigcup_{i=1}^{5} L_{i}$. Define $\underline{Z}_{\{k\}}=H\left(\underline{z}_{i j}\right)$, where $\underline{z}_{i j}$ is the path from point $i$ to point $j$, when the part of the path which is between $i$ and $j$ is below the $X$-axis, and it surrounds point $k$ from the left (if $k<i$ ) or from the right (if $j<k$ ). For example, see the following figures:

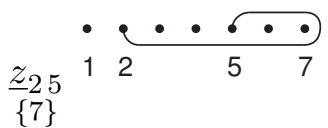




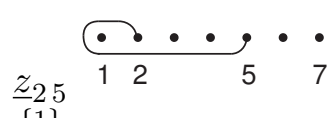

$\{1\}$

Corollary 2.3. After regenerating $V$ in a small neighborhood $U$ of $v, L_{4}$ turns into a conic $Q_{4}$, s.t. $Q_{4}$ is tangent to $L_{2}$ and $L_{5}$. Denote the resulting branch curve, after this regeneration, by $\tilde{V}$. Thus, the singularities of $T=\tilde{V} \cap U$ are as in the figure below:

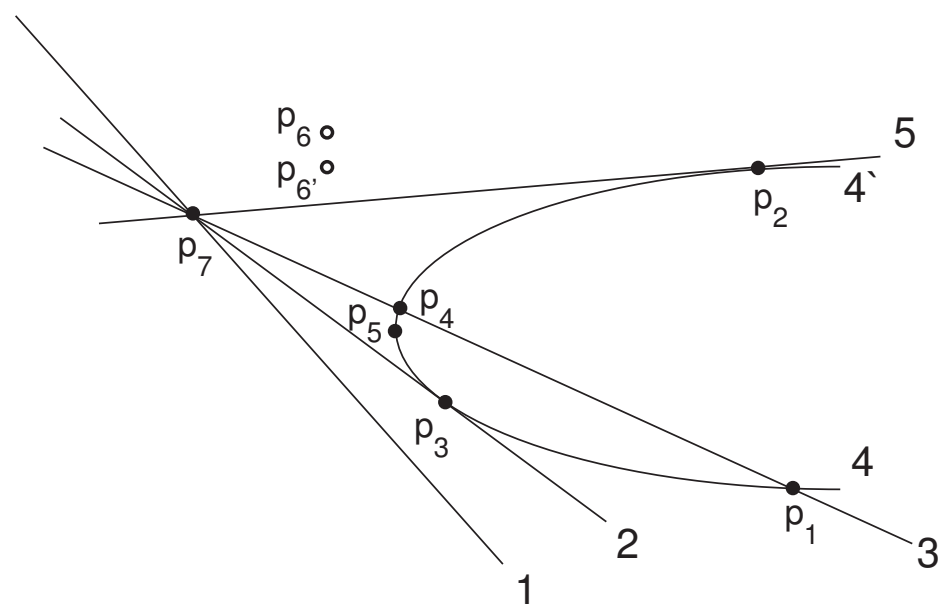

(figure 4)

Then the local braid monodromy of the above configuration is

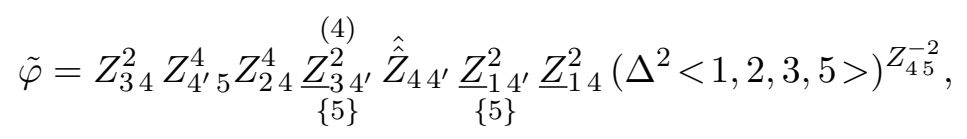

where $\hat{\hat{Z}}_{44^{\prime}}=H\left(\hat{\hat{z}}_{4,4^{\prime}}\right)\left(\underline{Z}_{\{5\}}^{2}=H\left(\underline{z}_{34}^{2}\right)\right)$ is the half-twist corresponding to the following path :

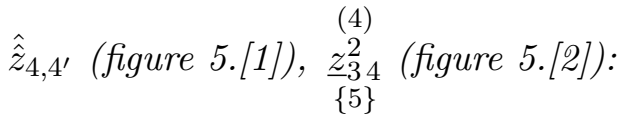

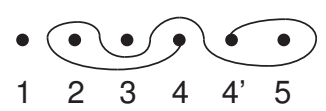

[1]

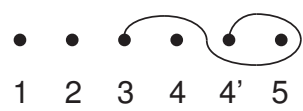

[2]

(figure 5) 
Proof: Let $\left\{p_{j}\right\}_{j=1}^{7} \cup\left\{p_{6^{\prime}}\right\}$ be the singular points of a small neighborhood (that is $-U$ ) of $v$ (see figure 4) with respect to $\pi_{1}$ (the projection to the $\mathrm{X}$-axis) as follows:

$p_{2}, p_{3}$ - the tangent points of $Q_{4}$ and $L_{2}, L_{5}$.

$\left\{p_{1}, p_{4}\right\},\left\{p_{6}, p_{6^{\prime}}\right\}$ - the intersection points of $Q_{4}$ with $L_{3}, L_{1}$.

$p_{5}$ - the branch point of $Q_{4}$.

$p_{7}$ - the intersection point of $\left\{L_{i}\right\}_{i=1,2,3,5}$.

Let $\mathrm{E}$ (resp. D) be a closed disk on the $X$-axis (resp. $Y$-axis). Let $N=\left\{x\left(p_{j}\right)=\right.$ $x_{j} \mid 1 \leq j \leq 7$ or $\left.j=6^{\prime}\right\}$, s.t. $N \subset E-\partial E$. Let $M$ be a real point on the $x$-axis, s.t. $x_{j} \ll M, \forall x_{j} \in N, 1 \leq j \leq 7$ or $j=6^{\prime}$. There is a $g$-base $\ell\left(\gamma_{j}\right)_{j=1}^{7} \cup \ell\left(\gamma_{6^{\prime}}\right)$ of $\pi_{1}(E-N, u)$, s.t. each path $\gamma_{j}$ is below the real line and the values of $\varphi_{M}$ with respect to this base and $E \times D$ are the ones given in the proposition. We look for $\varphi_{M}\left(\ell\left(\gamma_{j}\right)\right)$ for $j=1, \ldots, 7$ or $j=6^{\prime}$. Choose a $g$-base $\ell\left\{\gamma_{j}\right\}_{j=1}^{7} \cup \ell\left(\gamma_{6^{\prime}}\right)$ as above and put all the data in the following table:

\begin{tabular}{cccc}
$j$ & $\lambda_{x_{j}}$ & $\varepsilon_{x_{j}}$ & $\delta_{x_{j}}$ \\
\hline 1 & $<3,4>$ & 2 & $\Delta<3,4>$ \\
2 & $<4^{\prime}, 5>$ & 4 & $\Delta^{2}<4^{\prime}, 5>$ \\
3 & $<2,3>$ & 4 & $\Delta^{2}<2,3>$ \\
4 & $<4,4^{\prime}>$ & 2 & $\Delta<4,4^{\prime}>$ \\
5 & $<3,4>$ & 1 & $\Delta_{I R}^{\frac{1}{2}}<2>$ \\
$6,6^{\prime}$ & $<1,3>,<1,4>$ & 2 & $\Delta^{2}<1,3>$ \\
7 & $<1,2,4^{\prime}, 5>$ & 2 & -
\end{tabular}

Note: A short description of $\lambda_{x_{j}}, \varepsilon_{x_{j}}, \delta_{x_{j}}, \xi_{x_{j}}$ appears in section 2.1. For a full description and examples - see [6].

$\xi_{x_{1}}=z_{3,4}$

$\varphi_{M}\left(\ell\left(\gamma_{1}\right)\right)=Z_{3,4}^{2}$

$\xi_{x_{2}}=z_{4^{\prime}, 5}(\Delta<3,4>$ does not affect this path $)$

$\varphi_{M}\left(\ell\left(\gamma_{2}\right)\right)=Z_{4^{\prime}, 5}^{4}$

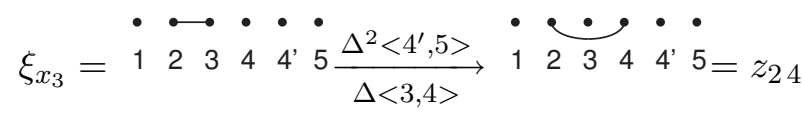

$\varphi_{M}\left(\ell\left(\gamma_{3}\right)\right)=Z_{24}^{4}$ 


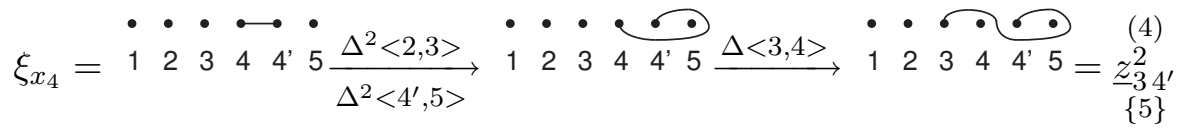

$$
\begin{aligned}
& \varphi_{M}\left(\ell\left(\gamma_{4}\right)\right)=\frac{(4)}{Z_{3}^{2} 4^{\prime}}
\end{aligned}
$$

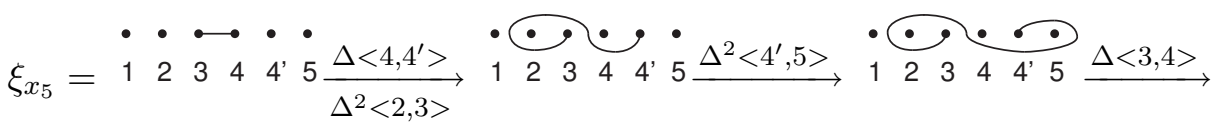

$$
\begin{aligned}
& \text { - } 20 \\
& \begin{array}{llllll}
12 & 3 & 4 & 4^{\prime} 5 & \hat{\hat{z}}_{44^{\prime}}
\end{array} \\
& \varphi_{M}\left(\ell\left(\gamma_{5}\right)\right)=\hat{\hat{Z}}_{44^{\prime}}
\end{aligned}
$$

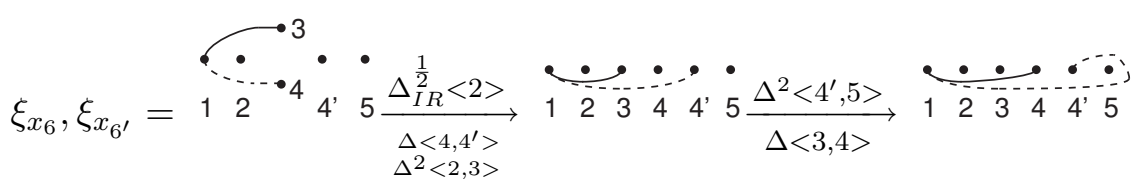

$$
\begin{aligned}
& \text { So }-\xi_{x_{6}^{\prime}}=\underline{z}_{14}, \xi_{x_{6}}=\underline{z}_{\{5\}} 4^{\prime}, \\
& \text { and by Corollary } 2.2-\varphi_{M}\left(\ell\left(\gamma_{6}\right) \ell\left(\gamma_{6^{\prime}}\right)\right)=\underline{Z}_{\{5\}}^{2} \underline{Z}_{14}^{2}
\end{aligned}
$$

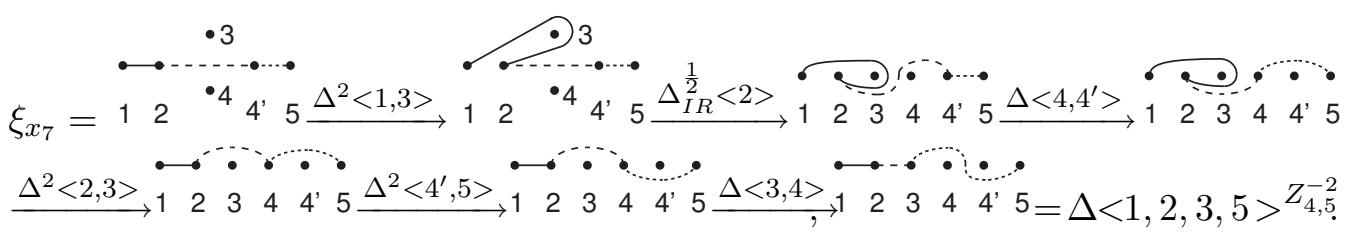

Note that in the first computation of $\xi_{x_{7}}$ we used Corollary 2.2; therefore, $\varphi_{M}\left(\ell\left(\gamma_{7}\right)\right)=\Delta^{2}<1,2,3,5>^{Z_{4,5}^{-2}}$.

The next relevant regeneration which affects the neighborhood of $v$ occurs when we regenerate lines 2 and 3. Note that in a small neighborhood of $\bigcup_{\substack{i=1,2 \\ 3,5}} L_{i}$ the regeneration process was already treated in [8], since the local configuration of the lines is as in figure 6 , and this is exactly the situation described in [8]. 


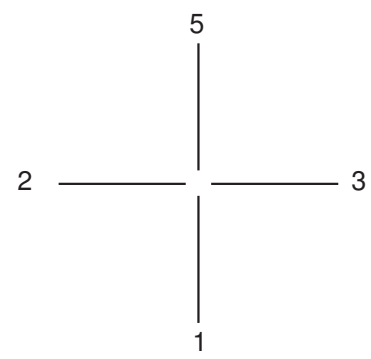

(figure 6)

Corollary 2.4. After the described regeneration, the local braid monodromy of a neighborhood of $v$ is given by-

$$
\tilde{\tilde{\varphi}}=Z_{34}^{2} Z_{4^{\prime}, 55^{\prime}}^{(3)} Z_{24}^{4} \underset{\left\{5^{\prime}\right\}}{\stackrel{(4)}{Z_{34^{\prime}}^{2}}} \hat{\hat{Z}}_{44^{\prime}} \underline{Z}_{\left\{5^{\prime}\right\}}^{2} \underline{Z}_{\left\{5^{\prime}\right\}}^{2} \underline{Z}_{1^{\prime} 4}^{2} \underline{Z}_{14}^{2}(B)^{Z_{45}^{-2} Z_{45^{\prime}}^{-2}}
$$

where $\hat{\hat{Z}}_{44^{\prime}}=H\left(\hat{\hat{z}}_{44^{\prime}}\right)$, and $\hat{\hat{z}}_{44^{\prime}}$ is the path represented by:

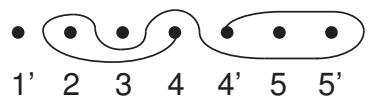

(figure 7)

and $B:=F(F)_{\rho^{-1}}$ where-

$F=Z_{1^{\prime}, 2}^{4} Z_{35}^{4} \tilde{Z}_{23} Z_{1^{\prime}, 5}^{2} \bar{Z}_{1^{\prime}, 5^{\prime}}^{2}$

$\rho=Z_{11^{\prime}} Z_{55^{\prime}}$ and $\tilde{Z}_{2,3}$ is represented by

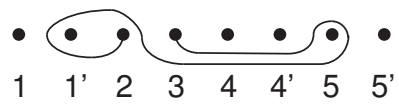

(figure 8)

Proof: After regenerating $\underset{\substack{i=1,2 \\ 3,5}}{\bigcup} L_{i}$ in a small neighborhood $U^{\prime}$ of $v, L_{2}$ and $L_{3}$ turn into conics: $Q_{2}, Q_{3}$ and $L_{1}, L_{5}$ double themselves into parallel lines $L_{1}, L_{1^{\prime}}, L_{5}, L_{5^{\prime}}$, s.t. $L_{1}, L_{1^{\prime}}$ is tangent to $Q_{3}$, and $L_{5}, L_{5^{\prime}}$ are tangent to $Q_{3}$. So by [8], Lemma 6 , when examining what happens in the process of the regeneration to $\Delta^{2}\langle 1,2,3,5\rangle$ (in $\tilde{\varphi}$ ), its local braid monodromy is $B$. Therefore, in the expression which represents the local braid monodromy of our current situation 
around $v, \Delta^{2}\langle 1,2,3,5\rangle$ is replaced by $B$. The other changes follow from the regeneration rules, as $L_{1}$ and $L_{5}$ are doubled. Therefore we get $\tilde{\tilde{\varphi}}$, by replacing in $\tilde{\varphi}$ the following:

(1) $Z_{4^{\prime} 5}^{4}$ into $Z_{4^{\prime}, 55^{\prime}}^{(3)}$ (third regeneration rule)

(2) $\underline{Z}_{1,4}^{2}$ into $\underline{Z}_{1^{\prime}, 4}^{2,5} \underline{Z}_{1,4}^{2}$ (second regeneration rule)

(3) $\underline{Z}_{1,4^{\prime}}^{2}$ into $\underline{Z}_{1^{\prime}, 4^{\prime}}^{2} \underline{Z}_{1,4^{\prime}}^{2}$ (second regeneration rule)

$\{5\} \quad\{5\} \quad\{5\}$

In the final regeneration that affects the neighborhood of $v$, the conics $Q_{2}, Q_{3}$ are doubled. Therefore, we have the following proposition:

Corollary 2.5. The local braid monodromy after the final regeneration around $v$ is given by

$$
\tilde{\tilde{\tilde{\varphi}}}=Z_{3^{\prime} 4}^{2} Z_{34}^{2} Z_{4^{\prime}, 55^{\prime}}^{(3)} Z_{22^{\prime}, 4}^{(3)} \frac{Z_{3^{\prime} 4^{\prime}}^{(4)}}{\left\{5^{\prime}\right\}}{\stackrel{Z}{Z_{3}^{\prime}}}_{\left\{5^{\prime}\right\}}^{(4)} \hat{\hat{Z}}_{44^{\prime}} \underline{Z}_{\left\{5^{\prime}\right\}}^{2}{\underline{L^{\prime} 4^{\prime}}}_{\left\{5^{\prime}\right\}}^{2} \underline{Z}_{1^{\prime} 4}^{2} \underline{Z}_{14}^{2}(\tilde{B})^{\bullet \bullet}
$$

where $\hat{\hat{Z}}_{44^{\prime}}$ corresponds to the path

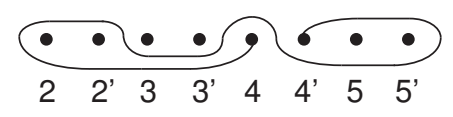

(figure 9)

( ) ${ }^{\bullet \bullet}$ is conjugation by $Z_{4,5}^{-2} Z_{4,5^{\prime}}^{-2}$

and $\tilde{B}=\tilde{F}(\tilde{F})_{\rho^{-1}}$ where

$\tilde{F}=Z_{1^{\prime}, 22^{\prime}}^{(3)} Z_{33^{\prime}, 5}^{(3)} \check{Z}_{2^{\prime} 3} \check{Z}_{23^{\prime}} \stackrel{\left(2-2^{\prime}\right)}{Z_{1^{\prime}, 5}^{2}} \bar{Z}_{1^{\prime}, 5^{\prime}}^{2}$

$\rho=Z_{11^{\prime}} Z_{55^{\prime}}$

and $\check{Z}_{23^{\prime}}, \check{Z}_{2^{\prime} 3}$ are:

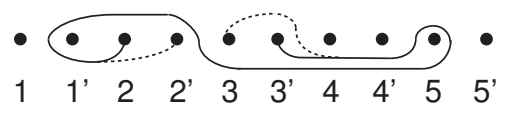

(figure 10)

Proof: All the changes follow from the regeneration rules.

We get $\tilde{\tilde{\varphi}}$ by replacing in $\tilde{\tilde{\varphi}}$ the following: 
(1) $Z_{3,4}^{2}\left(\stackrel{(4)}{\left(Z_{34^{\prime}}^{2}\right)} \underset{\left\{5^{\prime}\right\}}{\stackrel{(4)}{(4)}} \stackrel{(4)}{Z_{3^{\prime}, 4}^{2}} Z_{3,4}^{2}\right.$ (resp. $\left.\underline{Z}_{3^{\prime} 4^{\prime}}^{2} \underline{Z}_{34^{\prime}}^{2}\right)$ (second regeneration rule)
(2) $Z_{2,4}^{4}$ by $Z_{22^{\prime}, 4}^{(3)}$ (third regeneration rule); and we get $\tilde{B}(\tilde{F})$ by replacing in $B$ (resp. $F)$ the following:

(1) $Z_{1^{\prime}, 2}^{4}\left(Z_{3,5}^{4}\right)$ by $Z_{1^{\prime}, 22^{\prime}}^{(3)}$ (resp. $\left.Z_{33^{\prime}, 5}^{(3)}\right)$ (third regeneration rule)

(2) $\tilde{Z}_{2,3}$ by $\check{Z}_{2^{\prime} 3} \check{Z}_{23^{\prime}}$ (first regeneration rule)

2.3. The second case. The second case of the 5-point regeneration that we deal with is the braid monodromy factorization that we get from regenerating the following arrangement of 5 planes corresponding to the angular sectors of the figure:

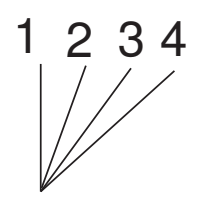

(figure 11)

when first we regenerate line number 4 , then line 3 , etc. But instead of looking at a particular case, we examine the general case where we have a line arrangement of $k$ lines, as in the following figure:

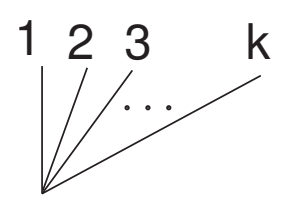

(figure 12)

when first we regenerate line number $k$ (i.e., $\ell_{k}$ ), then line $k-1$, etc. We denote $v$ to be the point of the intersection of all the lines.

When we regenerate $\ell_{k}$, this line turns into a conic $\tilde{p}_{k, k^{\prime}}$ (by Corollary 2.1) which is tangent to $\ell_{k-1}$. In a local neighborhood of $v$, the real part of this configuration of the lines $\ell_{1}, \ldots, \ell_{k-1}$ and the conic $\tilde{p}_{k, k^{\prime}}$ is as in the following figure: 


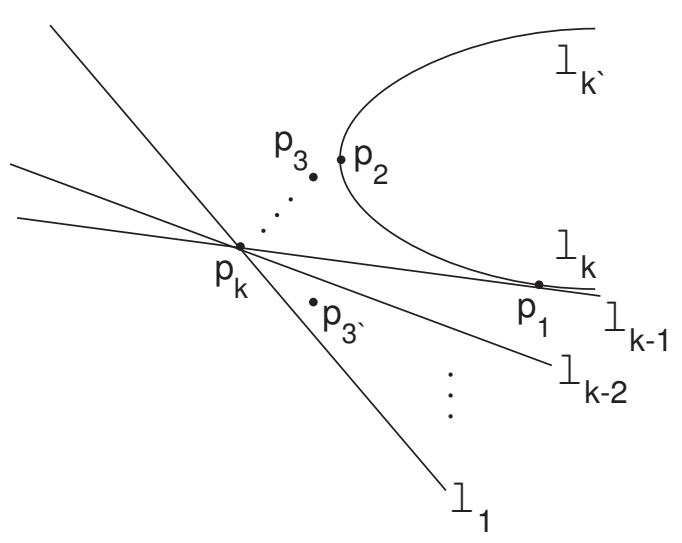

(figure 13)

Note that if we denote the slope of $\ell_{i}$ by $s_{i}$, then for two lines $-\ell_{i}, \ell_{j}(1 \leq i<$ $j \leq k-2$ and thus $\left.s_{i}<s_{j}\right)$ we have $\Re\left(\ell_{i} \cap \tilde{p}_{k, k^{\prime}}\right)<\Re\left(\ell_{j} \cap \tilde{p}_{k, k^{\prime}}\right)$.

We shall now compute the braid monodromy factorization of figure 13 .

Corollary 2.6. After regenerating $\ell_{k}$, the braid monodromy factorization in a local neighborhood of $v$ is:

$$
B_{k}=Z_{k-1, k}^{4} \bar{Z}_{k, k^{\prime}} \prod_{\{k-1\}}^{k}\left(Z_{i=3}^{2} Z_{k-i+1, k^{\prime}} Z_{k-i+1, k}^{2}\right) \Delta^{2}\langle 1, k-1\rangle
$$

Proof: After the regeneration, $\ell_{k}$ turns into a conic $\tilde{p}_{k, k^{\prime}}$ and thus, by figure 8 , we have the following singular points with respect to $\pi_{1}$ (the projection to the $\mathrm{X}$-axis):

$p_{1}$ : tangent point of $\ell_{k-1}$ and $\tilde{p}_{k, k^{\prime}}$.

$p_{2}$ : branch point of $\tilde{p}_{k, k^{\prime}}$.

$p_{i}, p_{i^{\prime}}: \forall 3 \leq i \leq k$ the (complex) intersection points (nodes) of $\ell_{k-i+1}$ and $\tilde{p}_{k, k^{\prime}}$.

$p_{k+1}$ : the intersection point of the lines $\ell_{1}, \ldots \ell_{k-1}$.

We condense the needed data in the following table: 


\begin{tabular}{cccc}
$j$ & $\lambda_{x_{j}}$ & $\varepsilon_{x_{j}}$ & $\delta_{x_{j}}$ \\
\hline 1 & $<k-1, k>$ & 4 & $\Delta^{2}<k-1, k>$ \\
2 & $<k, k^{\prime}>$ & 1 & $\Delta_{I R}<k-1>$ \\
$3,3^{\prime}$ & $<k-2, k>,<k-2, k^{\prime}>$ & 2 & $\Delta^{2}<k-2, k>$ \\
$4,4^{\prime}$ & $<k-3, k>,<k-3, k^{\prime}>$ & 2 & $\Delta^{2}<k-3, k>$ \\
$\vdots$ & $\vdots$ & $\vdots$ & $\vdots$ \\
$\mathrm{k}, \mathrm{k}$ & $<1, k>,<1, k^{\prime}>$ & 2 & $\Delta^{2}<1, k>$ \\
$\mathrm{k}+1$ & $<1, k-1>$ & 2 & -
\end{tabular}

Therefore

$\xi_{x_{1}}=z_{k-1, k}$

$\varphi_{M}\left(\ell\left(\gamma_{1}\right)\right)=Z_{k-1, k}^{4}$

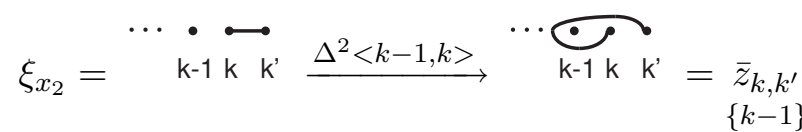

$\varphi_{M}\left(\ell\left(\gamma_{2}\right)\right)=\bar{Z}_{k, k^{\prime}}$

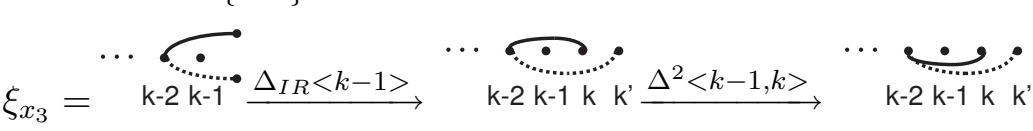

$\varphi_{M}\left(\ell\left(\gamma_{3}\right) \ell\left(\gamma_{3^{\prime}}\right)\right)=Z_{k-2, k^{\prime}}^{2} Z_{k-2, k}^{2}$

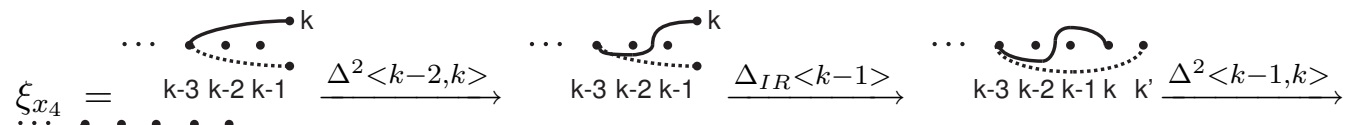
$k-3 \mathrm{k}-2 \mathrm{k}-1 \mathrm{k}$ k'

$\varphi_{M}\left(\ell\left(\gamma_{4}\right) \ell\left(\gamma_{4^{\prime}}\right)\right)=Z_{k-3, k^{\prime}}^{2} Z_{k-3, k}^{2}$.

Thus, for $3 \leq i \leq k$ :

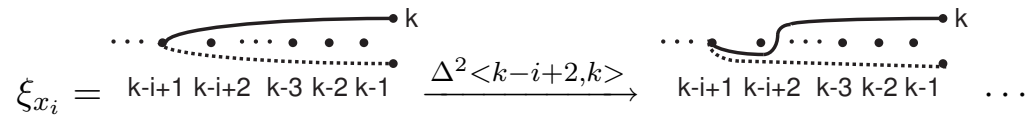
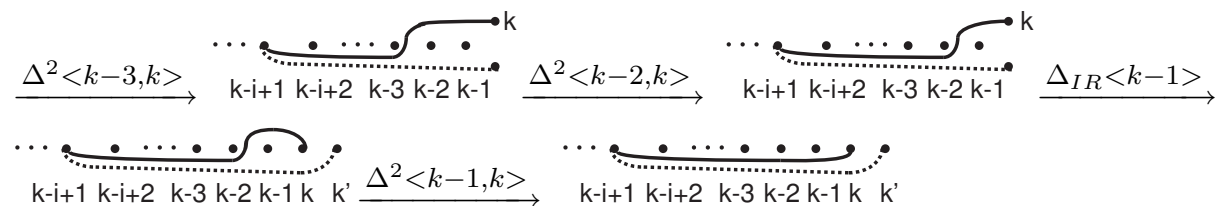

$\varphi_{M}\left(\ell\left(\gamma_{i}\right) \ell\left(\gamma_{i}^{\prime}\right)\right)=Z_{k-i+1, k^{\prime}}^{2} Z_{k-i+1, k}^{2}$ 


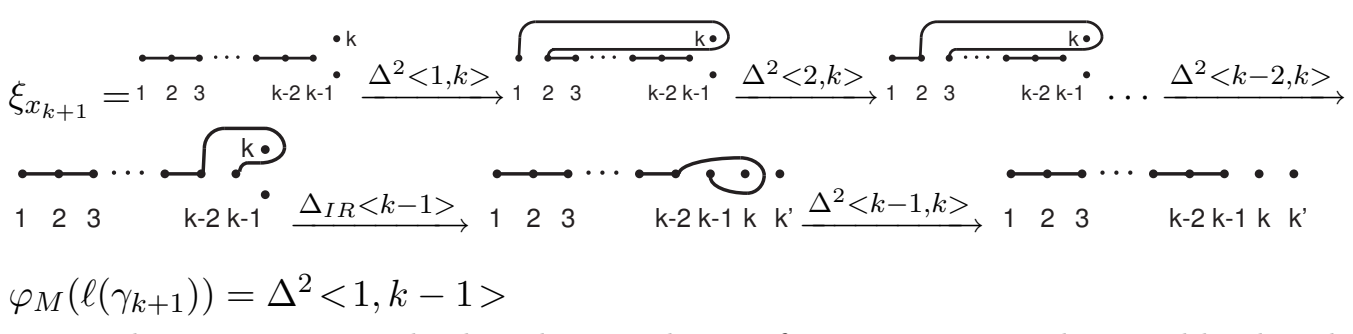

Note when computing the braid monodromy factorization in the neighborhood of the complex points we used Corollary 2.2.

The next step is to regenerate $\ell_{k-1}$ into a conic: $\tilde{p}_{(k-1),(k-1)^{\prime}}$. So we have the following:

Corollary 2.7. After the regeneration of $\ell_{k-1}$, the braid mondromy factorization in a local neighborhood of $v$ is:

$$
B_{k}^{(1)}=T_{k} \prod_{i=3}^{k}\left(Z_{k-i+1, k^{\prime}}^{2} Z_{k-i+1, k}^{2}\right) B_{k-1}
$$

where

$$
T_{k}=Z_{\left((k-1),(k-1)^{\prime}\right), k}^{(3)} \bar{Z}_{\{k-1\}}
$$

Proof: All the changes follow from the regeneration rules.

We get $B_{k}^{(1)}$ by replacing in $B_{k}$ the following:

(1) $Z_{k-1, k}^{4}$ by $Z_{\left((k-1),(k-1)^{\prime}\right), k}^{(3)}$ (by the third regeneration rule)

(2) $\Delta^{2}<1, k-1>$ by $B_{k-1}$ (This is implementation of corollary 2.6, where we have only $k-1$ lines).

The next step is the regeneration of $\ell_{k-2}$ (which turns into a conic $\left.\tilde{p}_{(k-2),(k-2)^{\prime}}\right)$.

So

Corollary 2.8. After the regeneration of $\ell_{k-2}$, the braid mondromy factorization in a local neighborhood of $v$ is:

$$
B_{k}^{(2)}=T_{k} \prod_{i=3}^{3} Z_{k-i+1,(k-i+1)^{\prime}, k, k^{\prime}}^{2} \prod_{i=4}^{k}\left(Z_{k-i+1, k^{\prime}}^{2} Z_{k-i+1, k}^{2}\right) B_{k-1}^{(1)}
$$

where

$$
Z_{\alpha, \alpha^{\prime}, \beta, \beta^{\prime}}^{2}=Z_{\alpha^{\prime}, \beta^{\prime}}^{2} Z_{\alpha^{\prime}, \beta}^{2} Z_{\alpha, \beta^{\prime}}^{2} Z_{\alpha, \beta}^{2}
$$


Proof: We get $B_{k}^{(2)}$ by replacing in $B_{k}^{(1)}$ the following:

(1) $Z_{k-2, k^{\prime}}^{2} Z_{k-2, k}^{2}$ by $Z_{k-2,(k-2)^{\prime}, k, k^{\prime}}^{2}$ (by the second regeneration rule)

(2) $B_{k-1}$ by $B_{k-1}^{(1)}$ (By implementation of corollary 2.7 ).

From now on, the braid monodromy factorization after regenerating $\ell_{k-3}$ (and then $\ell_{k-4}$ etc.) can be found by a recursive formula, as we apply the regeneration rules. Thus, the braid monodromy factorization after regenerating $\ell_{k-n}$ is

$$
B_{k}^{(n)}=T_{k} \prod_{i=3}^{n+1} Z_{k-i+1,(k-i+1)^{\prime}, k, k^{\prime}}^{2} \prod_{i=n+2}^{k}\left(Z_{k-i+1, k^{\prime}}^{2} Z_{k-i+1, k}^{2}\right) B_{k-1}^{(n-1)} .
$$

Naturally, the process ends when there are no lines to regenerate, that is, after the regeneration of $\ell_{1}$. For example, we examine the braid monodromy factorization of the 5 -point (when $k=4$ ):

$$
\begin{gathered}
\Delta^{2}<1,4>\stackrel{\text { regenerating } \ell_{4}}{\longrightarrow} B_{4}=Z_{3,4}^{4} \bar{Z}_{4,4^{\prime}} \prod_{i=3}^{4}\left(Z_{5-i, 4^{\prime}}^{2} Z_{5-i, 4}^{2} \Delta^{2}<1,3>\right. \\
\stackrel{\text { regenerating } \ell_{3}}{\longrightarrow} \\
B_{4}^{(1)}=T_{4} \prod_{i=3}^{4}\left(Z_{5-i, 4^{\prime}}^{2} Z_{5-i, 4}^{2}\right) Z_{2,3}^{4} \bar{Z}_{3,3^{\prime}} Z_{1,3^{\prime}}^{2} Z_{1,3}^{2} \Delta^{2}<1,2> \\
B_{4}^{(2)}=T_{4} Z_{2,2^{\prime}, 4,4^{\prime}}^{2} Z_{1,4^{\prime}}^{2} Z_{1,4}^{2} T_{3} Z_{1,3^{\prime}}^{2} Z_{1,3}^{2} Z_{1,2}^{4} \bar{Z}_{2,2^{\prime}} \\
\{1\} \\
\stackrel{\text { regenerating } \ell_{2}}{\longrightarrow} \\
B_{4}^{(3)}=T_{4} Z_{2,2^{\prime}, 4,4^{\prime}}^{2} Z_{1,1^{\prime}, 4,4^{\prime}}^{2} T_{3} Z_{1,1^{\prime}, 3,3^{\prime}}^{2} T_{2}
\end{gathered}
$$

Thus, $B_{5}^{(4)}$ is the braid monodromy factorization of the fully regenerated neighborhood of the 5-point. 
Remark: It is easy to prove (using the recursive formula) that the braid monodromy factorization of the fully regenerated neighborhood of the $k+1$-point in figure 12 is:

$$
B_{k}^{(k-1)}=\prod_{j=k}^{2}\left(T_{j} \prod_{m=j-2}^{1} Z_{m, m^{\prime}, j, j^{\prime}}^{2}\right)
$$

where

$$
T_{j}=Z_{\left((j-1),(j-1)^{\prime}\right), j}^{(3)} \bar{Z}_{j j-1\}}
$$

\section{USING THE 5-POINT REGENERATION: \\ THE HirzebruCH SURFACE $F_{2,(2,2)}$}

In this section we give an example of using the special braid monodromy factorization of the 5-point, described in subsection 2.2, in order to find the global braid monodromy factorization of the branch curve of a generic projection of $F_{2,(2,2) \text {. }}$

Remark: The second case of the regeneration (considered in subsection 2.3, for arbitrary $k$ ) appears when we compute, for example, the global braid monodromy factorization of the branch curve of a generic projection of $F_{k,(a, b)}$ when $k>2$. Note that finding the global braid monodromy of the Hirzebruch surface $F_{k,(a, b)}, \forall k, \forall a, b>1$ can be handled using only the classical 3- and 6-points, the 5 -point studied in subsection 2.2, and the $k+1$-point studied in subsection 2.3.

3.1. Braid monodromy of the degenerated curve. The configurations below describe the projective degeneration of $F_{2,(2,2)}=Z^{(0)} \rightsquigarrow Z^{(1)} \rightsquigarrow \ldots \rightsquigarrow Z^{(9)}$ 
$z^{(0)}$ $Z^{(1)}$
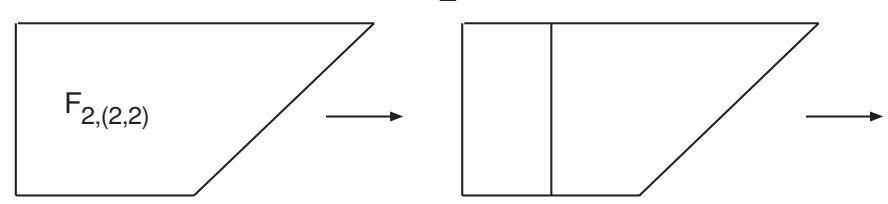

$z^{(2)}$ $Z^{(3)}$ $Z^{(4)}$

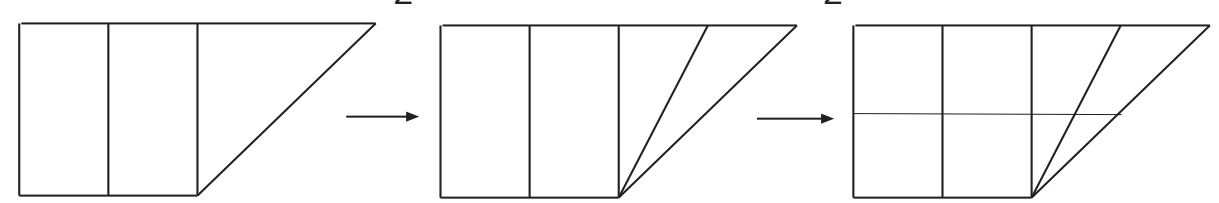

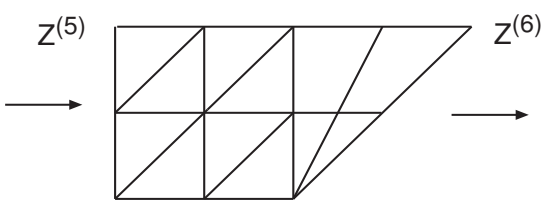

$Z^{(8)}$

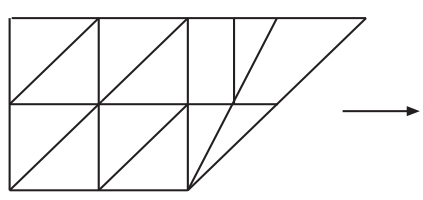

$Z^{(9)}$

$Z^{(7)}$
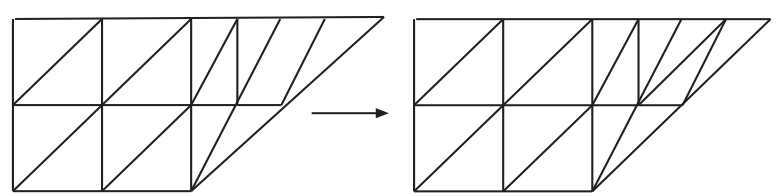

(figure 14)

So $F_{2,(2,2)}$ is degenerated into a union of 16 planes (see [4] for a detailed description of the degeneration of Hirzebruch surfaces), where the lines represent the intersection of the planes, and the order of the vertices is chosen to be lexicographic. See figure 15:

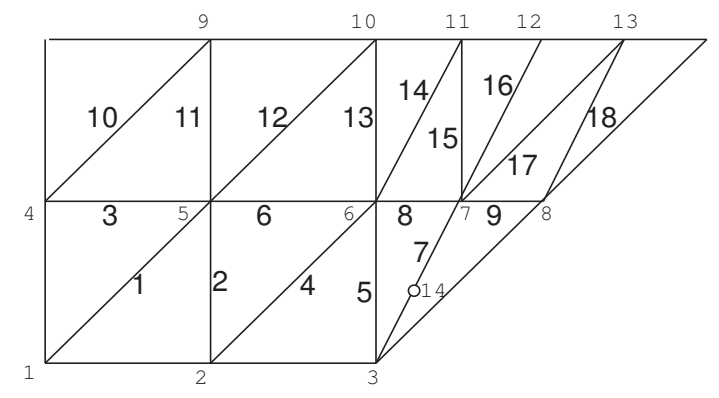

(figure 15) 
We denote the numeration of the intersection lines on $Z^{(9)}$ by $\left\{\widehat{L}_{i}\right\}_{i=1}^{18}$ by the following method: Let $\widehat{L_{1}}, \widehat{L_{2}}$ be two edges, where $\widehat{L_{i}}$ has vertices $\alpha_{i}<\beta_{i}$. Set $\widehat{L_{1}}<\widehat{L_{2}}$ iff $\beta_{1}<\beta_{2}$ or $\beta_{1}=\beta_{2}$ and $\alpha_{1}<\alpha_{2}$. Denote also by $\left\{\tilde{v}_{i}\right\}_{i=1}^{14}$ the intersection points. The appearance of the point numbered 14, which is an extra branch point, will be explained in subsection 3.2.5. Take a generic projection $\pi^{(i)}: Z^{(i)} \rightarrow \mathbb{C P}^{2}, 0 \leq i \leq 9$. Let $S^{(i)}$ be the branch curve in $\mathbb{C P}^{2}, \varphi^{(i)}$ their braid monodromy, $S^{(i+1)}$ be a degeneration of $S^{(i)}$ (for $0 \leq i \leq 9$ ). Let $L_{j}=$ $\pi^{(9)}\left(\widehat{L_{j}}\right), j=1 \ldots 18$. So $S^{(9)}=\bigcup_{j=1}^{18} L_{j}$; and $v_{j}=\pi^{(9)}\left(\widetilde{v_{j}}\right), j=1 \ldots 14$, so $v_{j}$ are the singular points of $S^{(9)}$. Let $C$ be the union of all lines connecting pairs of the $v_{j}$-s. $S^{(9)}$ is a subcurve of $C$. Theorem IX, 2.1, in [5] gives a full description of the braid monodromy of $C: \Delta_{C}^{2}=\prod_{i=1}^{14} C_{i} \Delta_{v_{i}}^{2}$ with an appropriate description of L.V.C. We use this formula to obtain a description of $\varphi^{(9)}$ by deleting all factors that involve lines which do not appear in $S^{(9)}$. Thus, we get $\Delta_{S^{(9)}}^{2}=\prod_{i=1}^{14} \widetilde{C}_{i} \tilde{\Delta}_{v_{i}}^{2}$. We describe each factor separately.

$\tilde{\Delta}_{v_{i}}^{2}$ : In $S^{(9)}$, we have 3 points which are 6 -point (points that arise from the intersection of 6 planes), which are $v_{5}, v_{6}, v_{7} ; 8$ points which are 3 -point, which are $\left\{v_{j}\right\}, \mathrm{j}=2,3,4,8,9,10,11,13$ and two points which are 2-point, which are $v_{1}, v_{12}$. We denote by $v_{14}$ the extra branch point, which appears during the regeneration of the line $L_{7}$ (see subsection 3.2.5). Since it contributes a factor to the final braid monodromy factorization, we mention now that the resulting braid monodromy factorization will be denoted as $\Delta^{2}=\prod_{i=1}^{14} C_{i}^{\prime} \varphi_{i}$.

The local braid monodromies $-\varphi_{j}^{(9)}$, which are $\tilde{\Delta}_{v_{i}}^{2}$, are introduced and regenerated in the following paragraphs.

$\widetilde{C_{i}}$ : We get 18 lines in $Z^{(10)}$. Each line $L_{i}$ is represented as a pair of its two end vertices. We define $L_{i}<L_{j}$ as above. Define $D_{t}=\prod_{\substack{p<t \\ L_{p} \cap L_{t}=\emptyset}} \tilde{Z}_{p t}^{2}$, where $\tilde{Z}_{p t}$ formulated in [5] (p. 526). $\tilde{Z}_{p t}^{2}$ are related to the parasitic intersections, since they are lines which do not intersect in $\mathbb{C P}^{15}$ but may intersect in $\mathbb{C P}^{2}$. Note that $1 \leq p, t \leq 13$, since we do not include $v_{14}$ in this calculation (see explanation for this in the following passage). Thus:

$D_{1}=D_{2}=D_{3}=D_{6}=i d, D_{4}=\bar{Z}_{14}^{2} \bar{Z}_{34}^{2}, D_{5}=\prod_{p=1}^{3} \bar{Z}_{\substack{p 5 \\(4)}}^{2}, D_{7}=\prod_{\substack{p=1 \\ p \neq 5}}^{6} \bar{Z}_{p 7}^{2}$, 
$D_{8}=\prod_{p=1}^{3} \bar{Z}_{p 8}^{2}, D_{9}=\prod_{p=1}^{6} \bar{Z}_{p 9}^{2}, D_{10}=\prod_{\substack{p=1 \\ p \neq 3}}^{9} \bar{Z}_{p 10}^{2}, D_{11}=\prod_{\substack{p=4 \\ p \neq 6}}^{9} \bar{Z}_{p 11}^{2}, D_{12}=\prod_{\substack{p=4 \\ p \neq 6}}^{10} \bar{Z}_{p 12}^{2}$,

$D_{13}=\prod_{\substack{p=1 . .3, 7,9 . .11}} \bar{Z}_{p, 13}^{2}, D_{14}=\prod_{\substack{p=1 . .3, 7,9.12}} \bar{Z}_{p, 14}^{2}, D_{15}=\prod_{\substack{p=1 . .6, 10 . .13}} \bar{Z}_{p 15}^{p \neq 1}, D_{16}=\prod_{\substack{p=1 . .6, 10 . .14}} \bar{Z}_{p, 16}^{2}$,

$D_{17}=\prod_{\substack{p=1 . .6, 10.14}} \bar{Z}_{p, 17}^{2}, D_{18}=\prod_{\substack{p=1 . .8, 10.16}} \bar{Z}_{p 18}^{2}$

defining $\tilde{C}_{j}=\prod_{V_{j} \in L_{t}} D_{t}$, where $V_{j}$ is the small vertex among the two vertices of $L_{t}$, and we get

$$
\begin{gathered}
\tilde{C}_{1}=i d, \tilde{C}_{2}=D_{4}, \tilde{C}_{3}=D_{5} \cdot D_{7}, \tilde{C}_{4}=D_{10}, \tilde{C}_{5}=D_{11} \cdot D_{12} \\
\tilde{C}_{6}=D_{8} \cdot D_{13} \cdot D_{14}, \tilde{C}_{7}=D_{9} \cdot D_{15} \cdot D_{16} \cdot D_{17}, \tilde{C}_{8}=D_{18} \\
\tilde{C}_{i}=i d, \text { where } i=9,10, \ldots, 13
\end{gathered}
$$

As was indicated, the factors $\tilde{C}_{j}$ correspond to parasitic intersections. For each point we examine the lines that go through it, and compute the parasitic intersections with the other lines. Since we have already looked at the lines passing through $v_{3}$, we can ignore the line on which the point $v_{14}$ lies (which is $L_{7}$ ), and by abuse of notation we denote $\tilde{C}_{14}=i d$.

\subsection{Local braid monodromy of the regenerated curve.}

3.2.1. Computation and regeneration of $\tilde{\Delta}_{v_{j}}^{2}$. We will deal with each type of point separately.

\subsubsection{The 3-point type.}

Corollary 3.1. The local braid monodromies $\varphi_{2}, \varphi_{3}, \varphi_{4}, \varphi_{8}, \varphi_{9}, \varphi_{10}, \varphi_{11}, \varphi_{13}$ are:

$$
\begin{array}{cl}
\varphi_{2}=Z_{22^{\prime}, 4}^{(3)} \cdot \tilde{Z}_{44^{\prime}(2)} & \varphi_{3}=Z_{55^{\prime}, 7}^{(3)} \cdot \tilde{Z}_{77^{\prime}(5)} \\
\varphi_{4}=Z_{33^{\prime}, 10}^{(3)} \cdot \tilde{Z}_{1010^{\prime}(3)} & \varphi_{8}=Z_{99^{\prime}, 18}^{(3)} \cdot \tilde{Z}_{1818^{\prime}(9)} \\
\varphi_{9}=Z_{10^{\prime}, 1111^{\prime}}^{(3)} \cdot \tilde{Z}_{1010^{\prime}(11)} & \varphi_{10}=Z_{12^{\prime}, 1313^{\prime}}^{(3)} \cdot \tilde{Z}_{1212^{\prime}(13)} \\
\varphi_{11}=Z_{14^{\prime}, 1515^{\prime}}^{(3)} \cdot \tilde{Z}_{1414^{\prime}(15)} & \varphi_{13}=Z_{17^{\prime}, 1818^{\prime}}^{(3)} \cdot \tilde{Z}_{1717^{\prime}(18)},
\end{array}
$$

where $\tilde{Z}_{i i^{\prime}(j)}=H\left(\tilde{z}_{i i^{\prime}(j)}\right)$, and $\tilde{z}_{i i^{\prime}(j)}$ is the following path: 
$\mathrm{i}<\mathrm{j}$

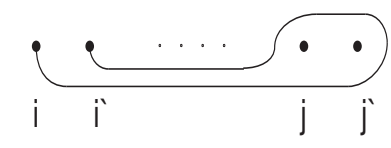

$i>j$

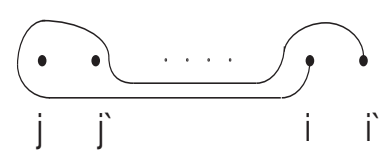

(figure 16)

Proof: See [8], lemma 1.

Remark: We will present the representing paths for the braid monodromy factorization for $\varphi_{2}$ (figures 17,18), $\varphi_{9}$ (figures 19,20). Note that this configuration of the paths is the same (with a suitable change of indices) for $\varphi_{3}, \varphi_{4}, \varphi_{8}$ (resp. $\left.\varphi_{10}, \varphi_{11}, \varphi_{13}\right)$ :

$Z_{22^{\prime}, 4}^{(3)}$ :

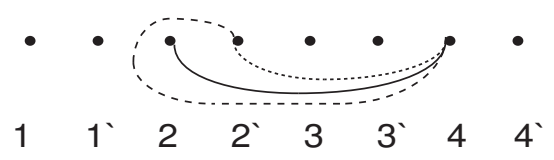

(figure 17)

$\tilde{Z}_{44^{\prime}(2)}$ :

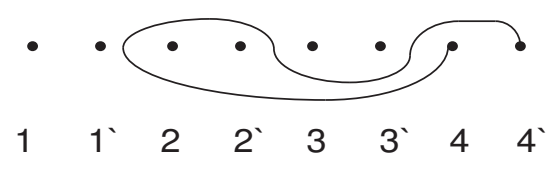

(figure 18)

$Z_{10^{\prime}, 1111^{\prime}}^{(3)}$ : 


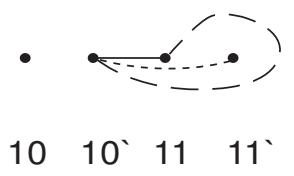

(figure 19)

$\tilde{Z}_{1010^{\prime}(11)}$

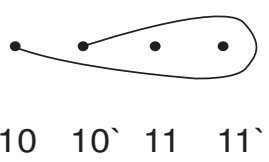

(figure 20)

3.2.3. The 6-point type. When regenerating $F_{2,(2,2)}$, a new kind of 6-point appears. Notice that the local numeration of the lines that intersect in $v_{5}, v_{6}$ is as follows:

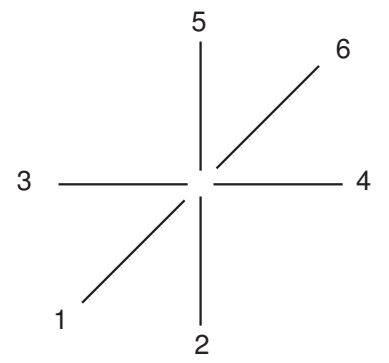

(figure 21)

We will call this kind of 6-point 6-PT1 (6-point type 1). Drawing (and numerating) the neighborhood of $v_{7}$ locally, we get: 


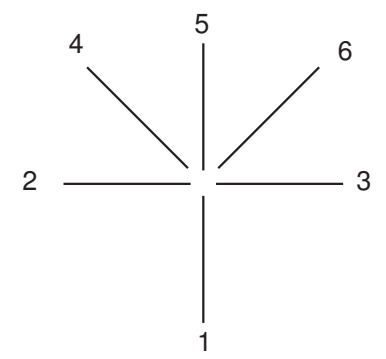

(figure 22)

This kind of 6-point will be called 6-PT2 (6-point type 2). We will deal first with the regeneration of $6-\mathrm{PT} 1$, which is more familiar, and then with 6-PT2.

\section{6-point type 1(6-PT1)}

Looking at figure 21, we see that this configuration of lines was already investigated in [8]. Therefore, we cite the main results from there:

Corollary 3.2. The local braid monodromies of $\varphi_{5}, \varphi_{6}$ are: $\varphi_{5}=Z_{1^{\prime}, 22^{\prime}}^{(3)} \tilde{Z}_{1212^{\prime}} Z_{33^{\prime}, 12^{\prime}}^{(2)}\left(Z_{22^{\prime}, 12^{\prime}}^{(2)}\right)^{\bullet} \bar{Z}_{66^{\prime}, 12}^{(3)}\left(Z_{33^{\prime}, 12}^{(2)}\right)^{\bullet}\left(Z_{22^{\prime}, 12}^{(2)}\right)^{\bullet}\left(\hat{F}_{5,1}\left(\hat{F}_{5,1}\right)_{\rho_{5}^{-1}}\right)^{\bullet} Z_{1111^{\prime}, 12}^{(3)}$

$$
\left(\prod_{\substack{i=12^{\prime}, 12,11^{\prime} \\ 11,6^{\prime}, 6}}\left(Z_{1^{\prime}, i}^{2}\right)\right)^{\bullet} \bar{Z}_{1^{\prime}, 33^{\prime}}^{(3)} \prod_{\substack{i=12^{\prime}, 12,11^{\prime} \\ 11,6^{\prime}, 6}}\left(Z_{1 i}^{2}\right) \tilde{Z}_{1,1^{\prime}},
$$

where ()$^{\bullet}$ is the conjugation by the braid induced from the motion:

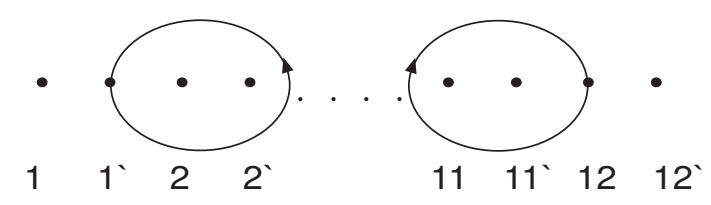

(figure 23)

and $\tilde{Z}_{11^{\prime}}, \tilde{Z}_{1212^{\prime}}$ are

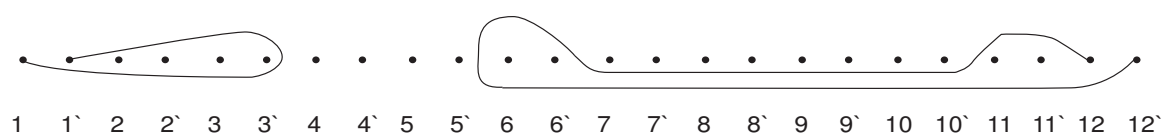

(figure 24) 
$\rho_{5}=Z_{22^{\prime}} Z_{1111^{\prime}}$

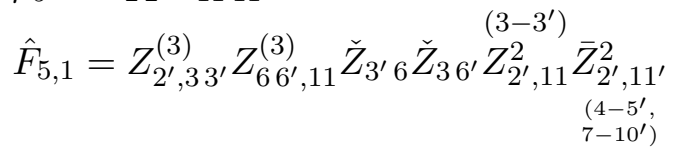

where $\check{Z}_{36^{\prime}}, \check{Z}_{3^{\prime} 6}$ are:

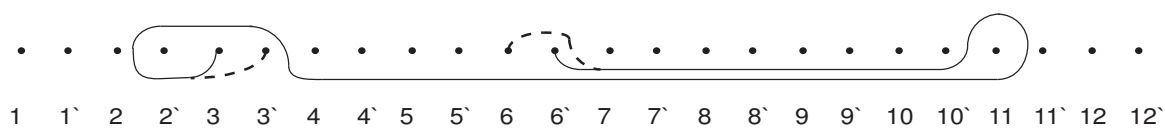

(figure 25)

$\varphi_{6}=Z_{4^{\prime}, 55^{\prime}}^{(3)} \tilde{Z}_{1414^{\prime}} Z_{66^{\prime}, 14^{\prime}}^{(2)}\left(Z_{55^{\prime}, 14^{\prime}}^{(2)}\right)^{\bullet \bullet} \bar{Z}_{88^{\prime}, 14}^{(3)}\left(Z_{66^{\prime}, 14}^{(2)}\right)^{\bullet \bullet}\left(Z_{55^{\prime}, 14}^{(2)}\right)^{\bullet \bullet}\left(\hat{F}_{6,1}\left(\hat{F}_{6,1}\right)_{\rho_{6}^{-1}}\right)^{\bullet \bullet}$

$$
Z_{1313^{\prime}, 14}^{(3)}\left(\prod_{\substack{i=14^{\prime}, 14,13^{\prime} \\ 13,8^{\prime}, 8}}\left(Z_{4^{\prime}, i}^{2}\right)\right)^{\bullet \bullet} \bar{Z}_{4^{\prime}, 66^{\prime}}^{(3)} \prod_{\substack{i=14^{\prime}, 11,13^{\prime} \\ 13,8^{\prime}, 8}}\left(Z_{4 i}^{2}\right) \tilde{Z}_{4,4^{\prime}}
$$

where ()$^{\bullet \bullet}$ is the conjugation by the braid induced from the motion:

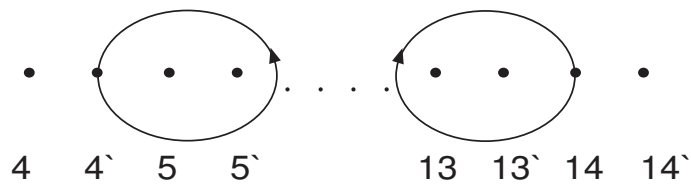

(figure 26)

and $\tilde{Z}_{44^{\prime}}, \tilde{Z}_{1414^{\prime}}$ are

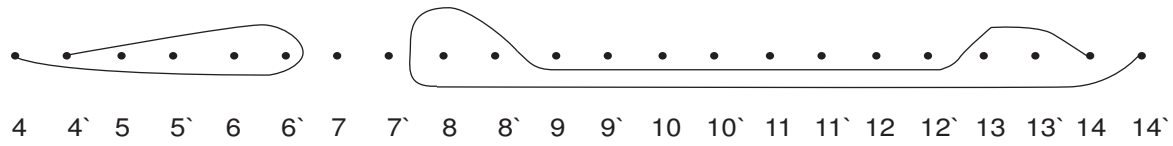

(figure 27)

$\rho_{6}=Z_{55^{\prime}} Z_{1313^{\prime}}$
$\hat{F}_{6,1}=Z_{5^{\prime}, 66^{\prime}}^{(3)} Z_{88^{\prime}, 13}^{(3)} \check{Z}_{6^{\prime} 8} \check{Z}_{68^{\prime}} Z_{5^{\prime}, 13}^{\left(6-6^{\prime}\right)} \bar{Z}_{5^{\prime}, 13^{\prime}}^{2}$
$\left(7-7^{\prime}\right.$
$\left.9-12^{\prime}\right)$

where $\check{Z}_{68^{\prime}}, \check{Z}_{6^{\prime} 8}$ are: 


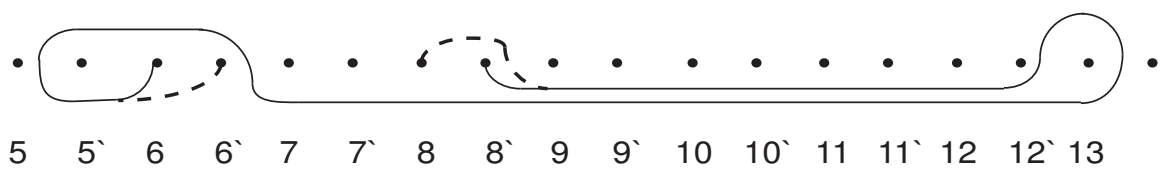

(figure 28)

\section{6-point type 2 (6-PT2)}

We are now dealing with the point $v_{7}$, that, when numerating the lines in a local neighborhood of $v_{7}, S^{(9)}$ is as in figure 24. The first regeneration that affects this neighborhood of $v_{7}$ is the regeneration from $Z^{(9)}$ to $Z^{(8)}$. The line $L_{6}$, is regenerated into a conic $Q_{6}$, that is tangent to $L_{3}$ and $L_{5}$. So, in a small neighborhood of $v_{7}, S^{(8)}$ is as in the following configuration:

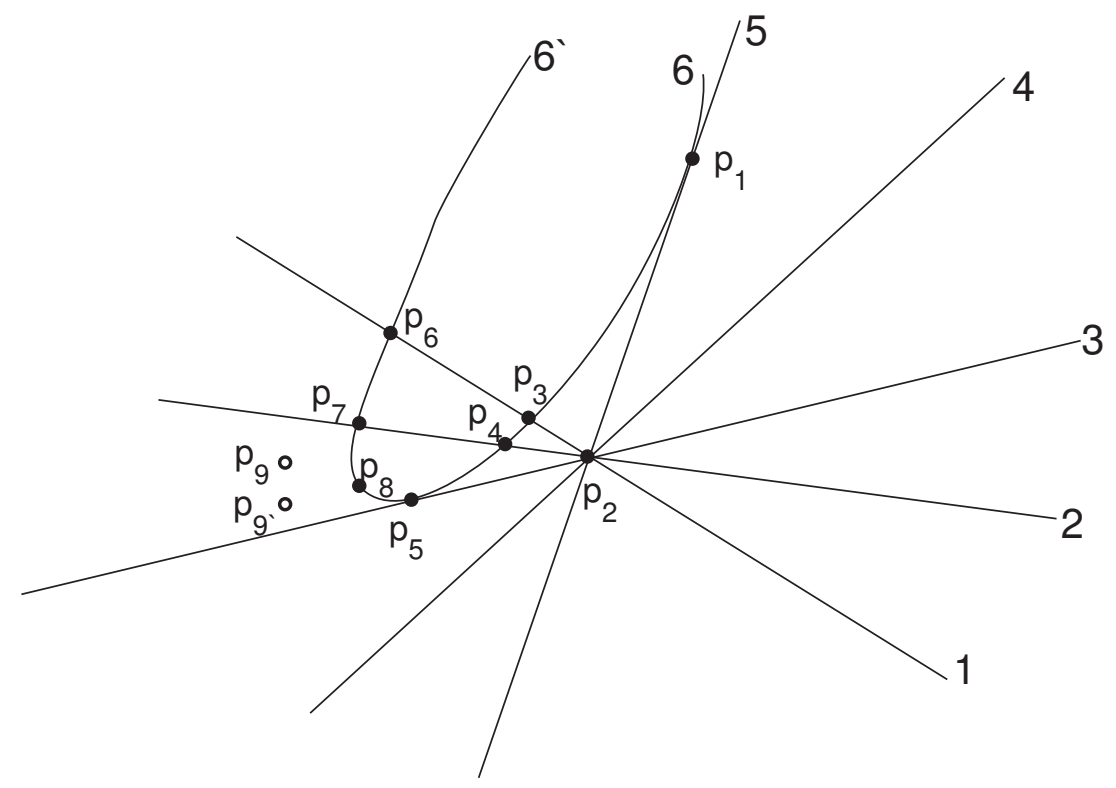

(figure 29)

As we can see, $L_{4}$ does not intersect the conic in the real part, so the intersection points of $L_{4}$ and $Q_{6}, p_{9}, p_{9^{\prime}} \in \mathbb{C}$. By looking at a particular model (where the conic is $\left.y^{2}=x, x_{p_{2}}>0\right)$, it is easy to see that $\Re\left(x_{p_{9}}\right)=\Re\left(x_{p_{9^{\prime}}}\right)<0$. So when calculating the braid monodromy factorization, we will use Corollary 2.2 .

Corollary 3.3. In a neighborhood of $v_{7}$, the local braid monodromy of $S^{(8)}$ around $v_{7}$ is given by 


$$
\varphi_{S^{(8)}}=Z_{5,6}^{4}\left(\Delta^{2}<1,5>\right)^{Z_{5,6}^{2}} \bar{Z}_{16}^{2} \bar{Z}_{26}^{2} \bar{Z}_{36}^{4} \bar{Z}_{16^{\prime}}^{2} \bar{Z}_{26^{\prime}}^{2} \tilde{Z}_{6,6^{\prime}} \tilde{Z}_{4,6^{\prime}}^{2} \underline{Z}_{4,6}^{2},
$$

where the path representing the braid $\tilde{Z}_{6,6^{\prime}}$ is:

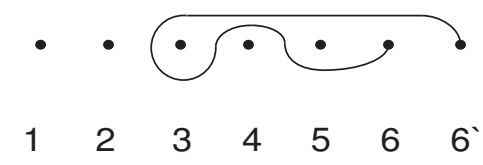

(figure 30)

and the path representing the braid $\tilde{\tilde{Z}}_{4,6^{\prime}}^{2}$ is

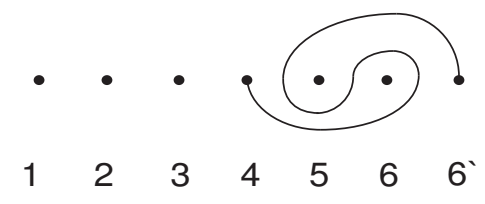

(figure 31)

Proof: Let $\left\{p_{j}\right\}_{j=1}^{9} \cup\left\{p_{9^{\prime}}\right\}$ be the singular points of a small neighborhood of $v_{7}$ (see figure 31 ) with respect to $\pi_{1}^{(8)}$ (the projection to the $\mathrm{X}$-axis) as follows: $p_{1}, p_{6}$ - tangent points of $Q_{6}$.

$\left\{p_{3}, p_{5}\right\},\left\{p_{4}, p_{7}\right\},\left\{p_{9}, p_{9^{\prime}}\right\}$ are the intersection points of $Q_{6}$ with $L_{1} / L_{2} / L_{4}$ (resp.). $p_{2}$ - an intersection point of $\left\{L_{i}\right\}_{i=1}^{5}$.

$p_{8}$ - the branch point of $Q_{6}$.

Let $\mathrm{E}$ (resp. D) be a closed disk on the $X$-axis (resp. $Y$-axis). Let $N=\left\{x\left(p_{j}\right)=\right.$ $x_{j} \mid 1 \leq j \leq 9$ or $\left.j=9^{\prime}\right\}$, s.t. $N \subset E-\partial E$. Let $M$ be a real point on the $x$-axis, s.t. $x_{j} \ll M, \forall x_{j} \in N, 1 \leq j \leq 9$ or $\left.j=9^{\prime}\right\}$. There is a $g$-base $\ell\left(\gamma_{j}\right)_{j=1}^{9,9^{\prime}}$ of $\pi_{1}(E-N, u)$, s.t. each path $\gamma_{j}$ is below the real line and the values of $\varphi_{M}$ w.r.t this base and $E \times D$ are the ones given in the proposition. We look for $\varphi_{M}\left(\ell\left(\gamma_{j}\right)\right)$ for $j=1, \ldots, 9,9^{\prime}$. Choose a $g$-base $\ell\left\{\gamma_{j}\right\}_{j=1}^{9,9^{\prime}}$ as above, and put all the data in the following table: 


\begin{tabular}{cccc}
$j$ & $\lambda_{x_{j}}$ & $\varepsilon_{x_{j}}$ & $\delta_{x_{j}}$ \\
\hline 1 & $<5,6>$ & 4 & $\Delta^{2}<5,6>$ \\
2 & $<1,5>$ & 2 & $\Delta<1,5>$ \\
3 & $<5,6>$ & 2 & $\Delta<5,6>$ \\
4 & $<4,5>$ & 2 & $\Delta<4,5>$ \\
5 & $<3,4>$ & 4 & $\Delta^{2}<3,4>$ \\
6 & $<6,6^{\prime}>$ & 2 & $\Delta<6,6^{\prime}>$ \\
7 & $<5,6>$ & 2 & $\Delta<5,6>$ \\
8 & $<4,5>$ & 1 & $\Delta_{I R}^{\frac{1}{2}}<3>$ \\
9 & $<2,6>$ & 2 & - \\
$9^{\prime}$ & $<2,6^{\prime}>$ & 2 & -
\end{tabular}

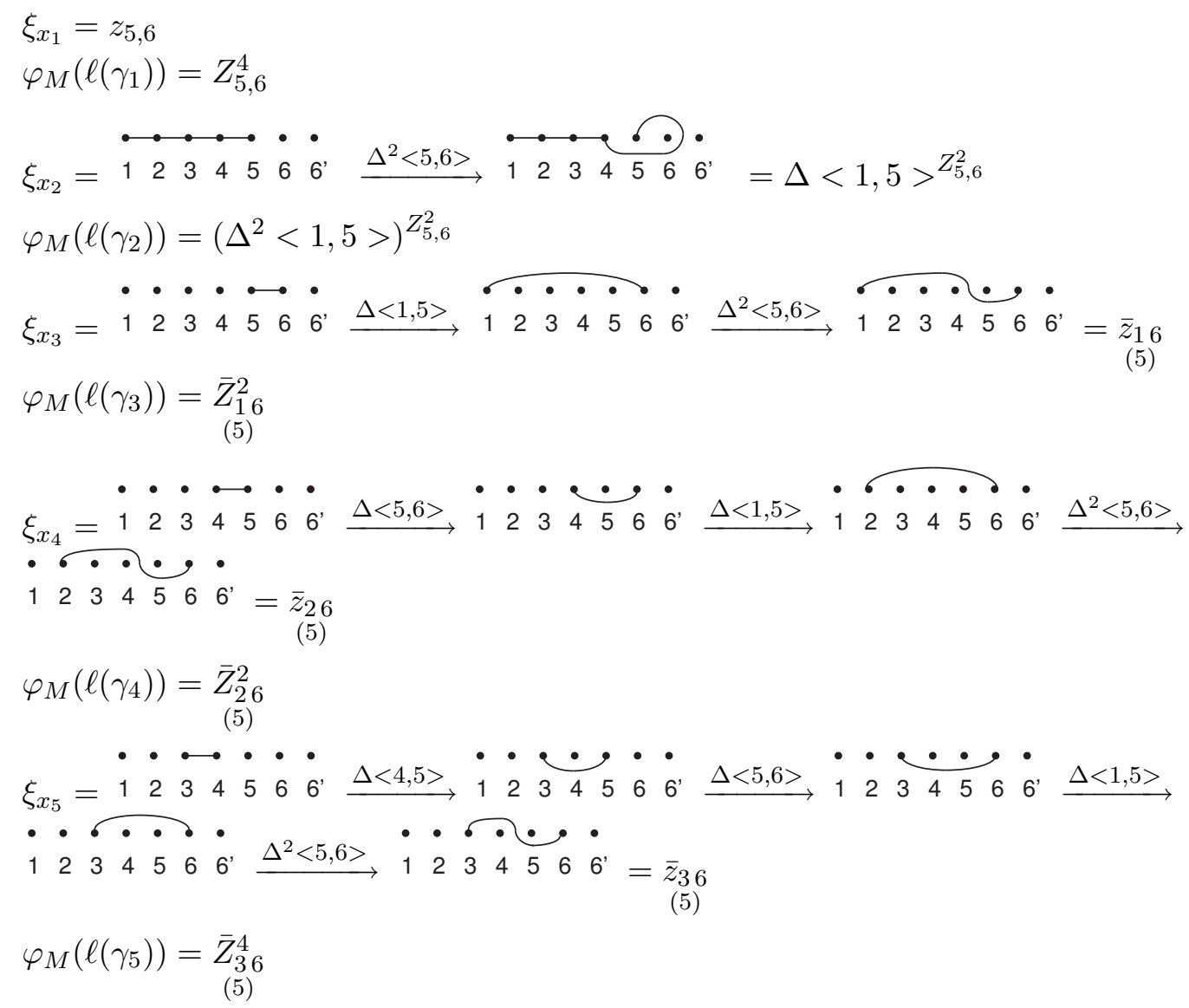




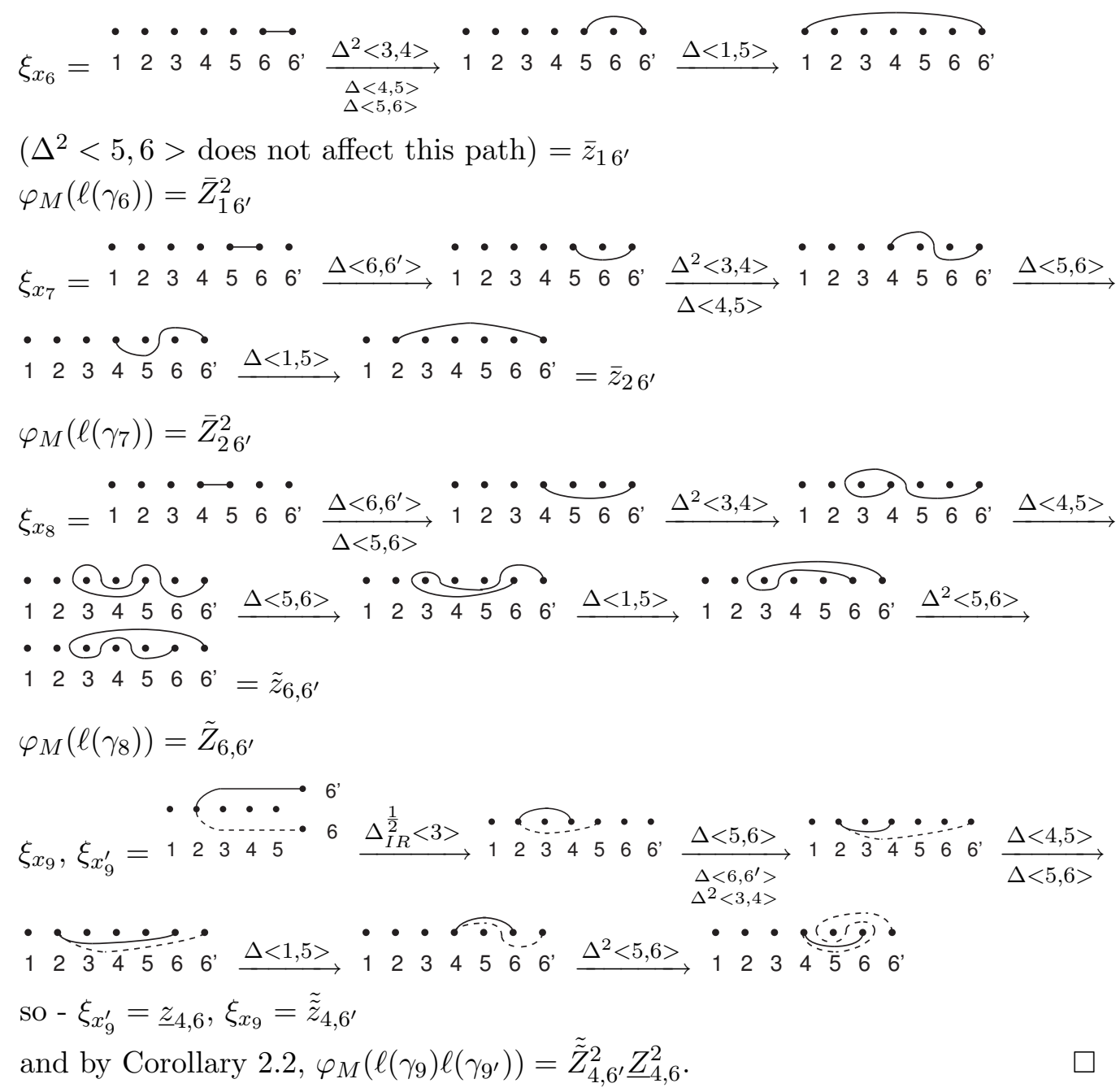

Note that we are now dealing with a situation described in the Section 2. That is, a local neighborhood of $v_{7}$ will look like figure 1 (in subsection 2.2). Therefore, we can use Corollary 2.5, which describes what happens to the braid monodromy factorization after all the regenerations (that is, the local braid monodromy of $\left.S^{(0)}\right)$. 
Corollary 3.4. The local braid monodromy of $S^{(0)}$ around $v_{7}$ is given by,

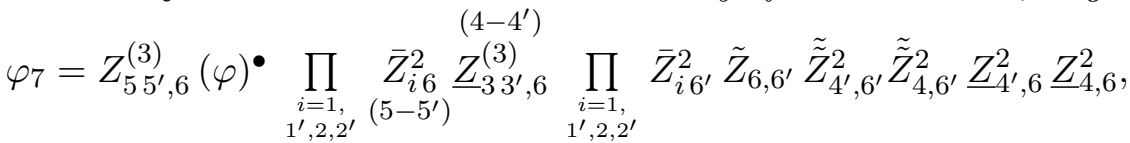
where $\tilde{\tilde{Z}}_{46^{\prime}}, \tilde{\tilde{Z}}_{4^{\prime} 6^{\prime}}$ are as in the following figure

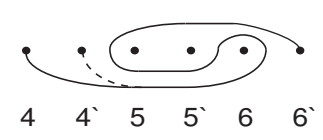

(figure 32)

$\tilde{Z}_{66^{\prime}}$ corresponds to the path:

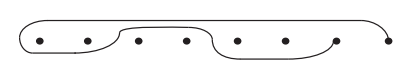

$\begin{array}{llllllll}3 & 3 & 4 & 4^{\prime} & 5 & 5^{\prime} & 6 & 6^{\prime}\end{array}$

(figure 33)

()$^{\bullet}$ is conjugation by $Z_{5^{\prime}, 6}^{2} Z_{5,6}^{2}$;

$\left(4-4^{\prime}\right)$

$\underline{Z}_{33^{\prime}, 6}^{(3)}$ is represented by the 3 following paths:

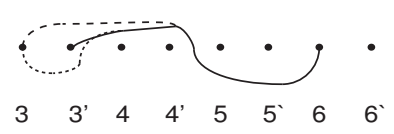

(figure 34)

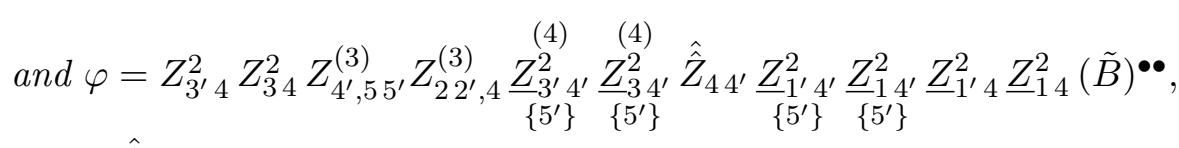

where $\hat{\hat{Z}}_{44^{\prime}}$ is as in Corollary 2.5, ( $)^{\bullet \bullet}$ is conjugation by $Z_{4,5}^{-2} Z_{4,5^{\prime}}^{-2}$ and $\tilde{B}=\tilde{F}(\tilde{F})_{\rho^{-1}}$ where

$\tilde{F}=Z_{1^{\prime}, 22^{\prime}}^{(3)} Z_{33^{\prime}, 5}^{(3)} \check{Z}_{2^{\prime} 3} \check{Z}_{23^{\prime}} \stackrel{\left(2-2^{\prime}\right)}{Z_{1^{\prime}, 5}^{2}} \bar{Z}_{1^{\prime}, 5^{\prime}}^{2}$

$\rho=Z_{11^{\prime}} Z_{55^{\prime}}$

and $\check{Z}_{23^{\prime}}, \check{Z}_{2^{\prime} 3}$ are as in Corollary 2.5.

Proof: All the changes follow from the regeneration rules and from Corollary 2.5. Thus, we get $\varphi_{7}$ by replacing in $\varphi_{S^{(8)}}$ the following: 
$\left(4-4^{\prime}\right)$

(1) By the third regeneration rule: $Z_{5,6}^{4} \rightarrow Z_{55^{\prime}, 6}^{(3)}, \bar{Z}_{36}^{4} \rightarrow \underline{Z}_{33^{\prime}, 6}^{(3)}$

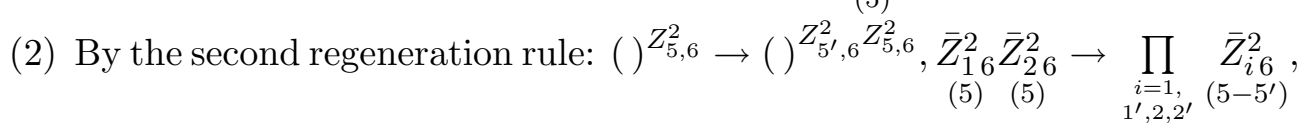
$\bar{Z}_{16^{\prime}}^{2} \bar{Z}_{26^{\prime}}^{2} \rightarrow \prod_{\substack{i=1, 1^{\prime}, 2,2^{\prime}}} \bar{Z}_{i 6^{\prime}}^{2}, \underline{Z}_{4,6}^{2} \rightarrow \underline{Z}_{4^{\prime}, 6}^{2} \underline{Z}_{4,6}^{2}, \tilde{\tilde{Z}}_{4,6^{\prime}}^{2} \rightarrow \tilde{\tilde{Z}}_{4^{\prime}, 6^{\prime}}^{2} \tilde{\tilde{Z}}_{4,6^{\prime}}^{2}$

(3) By Corollary 2.5: $\Delta^{2}<1,5>\rightarrow \varphi$.

Note: ()$^{\bullet \bullet},()^{\bullet}=$ conjugation by the braids induced from the motions:

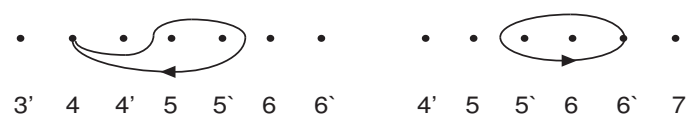

(figure 35)

Note: The above computation of $\varphi_{7}$ was done before the embedding of $B_{12}$ (the braid group with 12 strings, which in all of the above computation were done) into $B_{36}$ (the braid group with 36 strings, which in the braid monodromy factorization is expressed). So we have the following:

Remark: the local braid monodromy of $S^{(0)}$ around $v_{7}$, after embedding in $B_{36}$, is given by $\varphi_{7}=Z_{1616^{\prime}, 17}^{(3)}(\tilde{\varphi})^{\bullet} \prod_{\substack{i=7,\left(10-14^{\prime} \\ 7^{\prime}, 8,8^{\prime} \\ 16-16^{\prime}\right)}} \bar{Z}_{99^{\prime}, 17}^{2} \prod_{\substack{i=7,\left(15-15^{\prime}\right) \\ 7^{\prime}, 8,8^{\prime}}}^{(3)} \bar{Z}_{i 17^{\prime}}^{2} \tilde{Z}_{\left.17,14^{\prime}\right)} \tilde{\tilde{Z}}_{15^{\prime}, 17^{\prime}}^{2} \tilde{\tilde{Z}}_{15,17^{\prime}}^{2} \underline{Z}_{15^{\prime}, 17}^{2} \underline{Z}_{15,17}^{2}$ where $\tilde{\tilde{Z}}_{1517^{\prime}}, \tilde{\tilde{Z}}_{15^{\prime} 17^{\prime}}, \tilde{Z}_{1717^{\prime}}$ are as in the following figure:

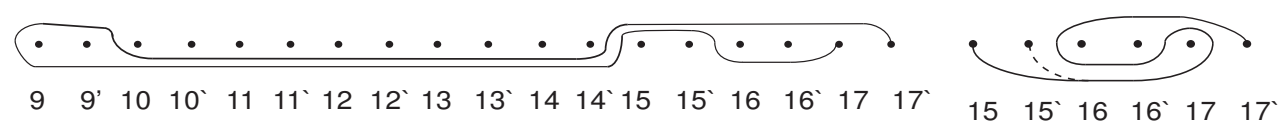

(figure 36)

()$^{\bullet}$ is conjugation by $Z_{16^{\prime}, 17}^{2} Z_{16,17}^{2}$; and

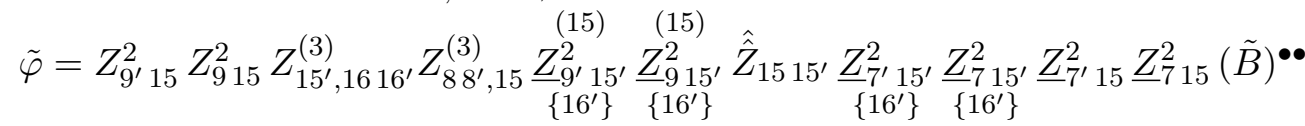

where $\hat{\hat{Z}}_{1515^{\prime}}$ corresponds to the path: 


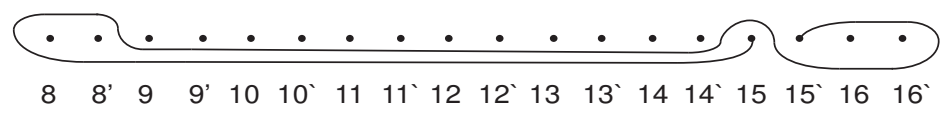

(figure 37)

( ) ${ }^{\bullet \bullet}$ is conjugation by $Z_{15,16}^{-2} Z_{15,16^{\prime}}^{-2}$ and

$\tilde{B}=\tilde{F}(\tilde{F})_{\rho^{-1}}$ where

$\tilde{F}=Z_{7^{\prime}, 88^{\prime}}^{(3)} Z_{99^{\prime}, 16}^{(3)} \check{Z}_{8^{\prime} 9} \check{Z}_{89^{\prime}} \stackrel{\left(8-8^{\prime}\right)}{Z_{7^{\prime}, 16}^{2}} \bar{Z}_{7^{\prime}, 16^{\prime}}^{2}$

$\rho=Z_{77^{\prime}} Z_{1616^{\prime}}$ and

$\check{Z}_{89^{\prime}}, \check{Z}_{8^{\prime} 9}$ are:

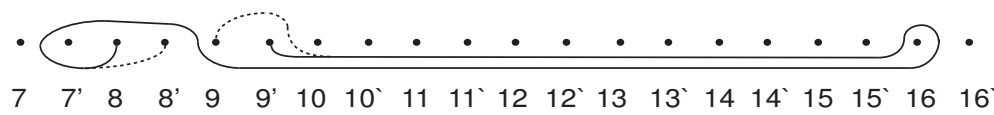

(figure 38)

3.2.4. Regeneration of $\tilde{C}_{i}$. Performing the regeneration affects also the parasitic line intersection. Denote by $C_{i}^{\prime}$ the braid, which is created from $\tilde{C}_{i}$ in the regeneration process. Every $\tilde{C}_{i}$ is a product of a 2-degree braid $\bar{Z}_{i j}^{2}$, which becomes, as a consequence of the second regeneration rule (see also [6], Proposition 3.2), an 8-degree braid: $\bar{Z}_{i i^{\prime}, j j^{\prime}}^{2}$ (where we denote by $\bar{Z}_{i i^{\prime}, j j^{\prime}}^{2}=Z_{i j}^{2} Z_{i^{\prime} j}^{2} Z_{i j^{\prime}}^{2} Z_{i^{\prime} j^{\prime}}^{2}$ ). So

$C_{i}^{\prime}=i d$, where $i=1,3^{\prime}, 9,10, \ldots, 13, C_{2}^{\prime}=D_{4}^{\prime}, C_{3}^{\prime}=D_{5}^{\prime} D_{7}^{\prime}, C_{4}^{\prime}=D_{10}^{\prime}$, $C_{5}^{\prime}=D_{11}^{\prime} D_{12}^{\prime}, C_{6}^{\prime}=D_{8}^{\prime} D_{13}^{\prime} D_{14}^{\prime}, C_{7}^{\prime}=D_{9}^{\prime} D_{15}^{\prime} D_{16}^{\prime} D_{17}^{\prime}, C_{8}^{\prime}=D_{18}^{\prime}$.

where

$$
\begin{aligned}
& D_{4}^{\prime}=\bar{Z}_{11^{\prime}, 44^{\prime}}^{2} \bar{Z}_{33^{\prime}, 44^{\prime}}^{2}, D_{5}^{\prime}=\prod_{p=1}^{3} \bar{Z}_{\substack{p p^{\prime}, 55^{\prime} \\
\left(4-4^{\prime}\right)}}^{2}, D_{7}^{\prime}=\prod_{\substack{p=1 \\
p \neq 5}}^{6} \bar{Z}_{p p^{\prime}, 77^{\prime}}^{2} \\
& D_{8}^{\prime}=\prod_{p=1}^{3} \bar{Z}_{\substack{p p^{\prime}, 88^{\prime} \\
\left(7-7^{\prime}\right)}}^{2}, D_{9}^{\prime}=\prod_{p=1}^{6} \bar{Z}_{p p^{\prime}, 99^{\prime}}^{2}, D_{10}^{\prime}=\prod_{\substack{p=1 \\
p \neq 3}}^{9} \bar{Z}_{p p^{\prime}, 1010^{\prime}}^{2}, D_{11}^{\prime}=\prod_{\substack{p=4 \\
p \neq 6}}^{9} \bar{Z}_{p p^{\prime}, 1111^{\prime}}^{2}\left(10-10^{\prime}\right) \\
& D_{12}^{\prime}=\prod_{\substack{p=4 \\
p \neq 6}}^{10} \bar{Z}_{p p^{\prime}, 1212^{\prime}}^{2}, D_{13}^{\prime}=\prod_{\substack{p=1 . .3, 7,9.11}} \bar{Z}_{p p^{\prime}, 1313^{\prime}}^{2}, \\
& D_{14}^{\prime}=\prod_{\substack{p=1 . .3, 7,9.12}} \bar{Z}_{p p^{\prime}, 1414^{\prime}}^{2}, D_{15}^{\prime}=\prod_{\substack{p=1 . .6, 10.13}} \bar{Z}_{p p^{\prime}, 1515^{\prime}}^{2}, \\
& D_{16}^{\prime}=\prod_{\substack{p=1 . .6, 10 . .14}} \bar{Z}_{p p^{\prime}, 1616^{\prime}}^{2}, D_{17}^{\prime}=\prod_{\substack{p=1 . .6, 10 . .14}} \bar{Z}_{p p^{\prime}, 1717^{\prime}}^{2}, D_{18}^{\prime}=\prod_{\substack{p=1 . .8, 10.16}} \bar{Z}_{p p^{\prime}, 1818^{\prime}}^{2}
\end{aligned}
$$


3.2.5. The 2-point type and extra branch points. There are two points $v_{1}, v_{12}$ which are 2-point. The regeneration around the point $v_{1}$ yields a conic $Q_{1}$ (resp. $\left.Q_{16}\right)$; that is because the pair of the two planes, that on their intersection the point $v_{1}$ (resp., and looking locally on the neighborhood of $v_{12}$ ) lies, is created in the degeneration process from a projective nonsingular quadric. But, in order that these 2-points will contribute a factor to the braid monodromy factorization, they should satisfy the condition of "Extra branch points", as described in [11], Section 4 . However, only $v_{1}$ satisfies this condition. Thus $v_{12}$ does not contribute a factor to this factorization (note that one can check this condition only after calculating the local factorization around $v_{7}$ ). By Artin's theorem, the induced braid monodromy in the neighborhood of $v_{1}$ is $Z_{11^{\prime}}$, namely, a counterclockwise halftwist of $1,1^{\prime}$. Thus, the local braid monodromy around $v_{1}$ is $\varphi_{1}=Z_{1,1^{\prime}}$.

However, there is another point $v_{14}$ (which is not a 2-point) that is an extra branch point which is created from the regeneration of the line $L_{7}$. This extra branch point contributes to our factorization the factor $Z_{7,7^{\prime}}$.

We will now prove the mentioned statements, following the ideas presented in [11]; we also follow its method of numeration, such that the numeration of the extra branch point is done last. Let us denote by $\tilde{\Delta}=\prod_{i=1}^{13} C_{i}^{\prime} \varphi_{i}$. If $\tilde{\Delta}$ is a braid monodromy factorization, then $\tilde{\Delta}=\Delta^{2}$ and $\operatorname{deg}(\tilde{\Delta})=36 \cdot(36-1)=1260$. However, $\operatorname{deg}(\tilde{\Delta})=1259$ (when assuming that $\varphi_{1}=Z_{1,1^{\prime}}, \varphi_{12}=i d$ as will be proved in the following lemma; the explicit calculation is made in subsection 3.3). This, there is a missing factor of degree 1 .

Define the forgetting homomorphisms:

$$
f_{i}: B_{36}\left[D,\left\{1,1^{\prime}, \ldots, 18,18^{\prime}\right\}\right] \rightarrow B_{2}\left[D,\left\{i, i^{\prime}\right\}\right], 1 \leq i \leq 18
$$

It is clear that $\forall i, \operatorname{deg}\left(f_{i}\left(\Delta^{2}\right)\right)=2$.

Lemma 3.1. (1) There exists an extra branch point $v_{14}$ that contributes a factor $\varphi_{14}=Z_{7,7^{\prime}}$ to the braid monodromy factorization.

(2) The regeneration of neighborhood of the point $v_{12}$ does not contribute a factor to braid monodromy factorization.

(3) The regeneration of neighborhood of the point $v_{1}$ does contribute a factor $\varphi_{1}=Z_{1,1^{\prime}}$ to braid monodromy factorization. 
Proof: (1) By Lemma 3.3.3 (or Proposition 3.3.4) in [10] (see also [11], Section 4 ), it is enough to prove that $\operatorname{deg}\left(f_{7}(\tilde{\Delta})\right)=1$. The braids coming from the parasitic intersections are sent by $f_{7}$ (and by any $f_{i}$, in fact) to $i d$, so it is enough to look only at the factors $\varphi_{j}, j \in\{1, \ldots, 13\}, j \neq 1,12$ that involve braids one of whose end points are 7 or $7^{\prime}$ (we omit the factors $\varphi_{1}, \varphi_{12}$ since we do not know yet what are they. In any case, they do not affect the result, since the do not fulfill the condition mentioned). The only suitable $j$ 's are $j=3$ and $j=7$. Since $v_{3}$ is a 3 -point, by Lemma 2, [8], $\operatorname{deg}\left(f_{7}\left(\varphi_{3}\right)\right)=1$. Examining $\varphi_{7}$, it is easy to see that there exists no braid in the factorization of $\varphi_{7}$ that is not sent to $i d$ by $f_{7} ;$ Thus $\operatorname{deg}\left(f_{7}\left(\varphi_{7}\right)\right)=0$. Therefore $\operatorname{deg}\left(f_{7}(\tilde{\Delta})\right)=1$.

(2) In order that the regeneration of the point $v_{12}$ will not contribute a factor to the braid monodromy factorization, we note that $v_{12}$ is only on the line $L_{16}$. So we actually have to check if $\sum_{\substack{i=1 \\ i \neq 12,1}}^{13} \operatorname{deg}\left(f_{16}\left(\varphi_{i}\right)\right)=2$ to prove the lemma (again, the braids coming from the parasitic intersections are sent to $i d$ by $f_{16}$ ). Examining $\varphi_{7}$, and using Lemma 2,[8], we get that $\operatorname{deg}\left(f_{16}\left(\varphi_{i}\right)\right)=2$ (since $\left.\operatorname{deg}\left(f_{16}\left(Z_{1616^{\prime}, 17}^{(3)}\right)\right)=\operatorname{deg}\left(f_{16}\left(\left(Z_{15^{\prime}, 1616^{\prime}}^{(3)}\right)\right)^{Z_{16^{\prime}, 17}^{2} Z_{16,17}^{2}}\right)=1\right)$, where $\forall 1 \leq i \leq 13, i \neq 1,7,12, \operatorname{deg}\left(f_{16}\left(\varphi_{i}\right)\right)=0$.

(3) This is done using the same method as in (2), by confirming that $\sum_{i=2}^{13} \operatorname{deg}\left(f_{1}\left(\varphi_{i}\right)\right)=1$.

\subsection{Global braid monodromy of the regenerated curve.}

Corollary 3.5. $\Delta_{36}^{2}=\prod_{i=1}^{14} C_{i}^{\prime} \tilde{\varphi}_{i}$ is a braid monodromy factorization for $S^{(0)}$, where $\tilde{\varphi}_{i}=\left(\varphi_{i}\right)_{h_{i}}$ for some $h_{i} \in\left\langle Z_{j j^{\prime}} \mid v_{i} \in L_{j}\right\rangle$.

Proof: Using Proposition VI.2.1 from [5] on $S^{(0)}$, we get that $\Delta_{36}^{2}=\prod_{i=1}^{14} C_{i}^{\prime} \tilde{\varphi}_{i} \prod b_{i}$, for some $h_{i} \in\left\langle Z_{j j^{\prime}} \mid v_{i} \in L_{j}\right\rangle$ determined by the regeneration of the embedding $B_{k} \hookrightarrow B_{18}$ to $B_{2 k} \hookrightarrow B_{36}$, where $k=1$ when $i=1,14, k=2$ when $i=2,3,4,8,9,10,11,13$, and $k=6$ if $k=5,6,7$ (see the definition of regeneration of an embedding in [8], section 1$)$. And where $b_{i}$ are factors corresponding to singularities that are not covered by $\prod C_{i}^{\prime} \tilde{\varphi}_{i}$, and each $b_{i}$ is of the form $Y_{i}^{t_{i}}, Y_{i}$ is a positive halftwist, $0 \leq t_{i} \leq 3$. Note that $\operatorname{deg}\left(\tilde{\varphi}_{i}\right)=\operatorname{deg}\left(\varphi_{i}\right)$. 
We shall compute $\operatorname{deg}\left(\prod_{i=1}^{14} C_{i}^{\prime} \varphi_{i}\right)=\sum^{14}\left(\operatorname{deg}\left(C_{i}^{\prime}\right)+\operatorname{deg}\left(\varphi_{i}\right)\right)$. So, $\sum^{14} \operatorname{deg}\left(C_{i}^{\prime}\right)=800$ (there are 100 factors; each factor - $Z_{i i^{\prime}, j j^{\prime}}^{2}$ - has degree 8).

For $i=2,3,4,8,9,10,11,13-v_{i}$ are 3 -point (in $S^{(9)}$ ); if $v_{i}$ is a 3 -point, then $\operatorname{deg}\left(\varphi_{i}\right)=10$ (by corollary 3.1 ); then $\sum_{\substack{i ; v_{i} i s \\ 3-\text { point }}} \operatorname{deg}\left(\varphi_{i}\right)=8 \cdot 10=80$.

For $i=1, v_{i}$ is 2-point; the line $L_{7}$ has an extra branch point (that is - in these cases we have a contribution of a factor of the form $Z_{i, i^{\prime}}$ ), so by the description in section 2.1, these factors contribute to the sum of degrees the addend 2 .

For $i=5,6,7, v_{i}$ is 6 -PT1 (for $i=5,6$ ) or a 6 -PT2 (for $i=7$ ). In any case, $\varphi_{i}$ includes:

6 factors with degree $1 \Rightarrow$ degree $=6$,

24 factors with degree $2 \Rightarrow$ degree $=48$,

24 factors with degree $3 \Rightarrow$ degree $=72$.

So, for a 6-point $v_{i}, \operatorname{deg}\left(\varphi_{i}\right)=126$ and $\sum_{\substack{i ; v_{i} i s \\ 6-\text { oint }}} \operatorname{deg}\left(\varphi_{i}\right)=3 \cdot 126=378$.

Therefore $-\operatorname{deg}\left(\prod_{i=1}^{14} C_{i}^{\prime} \varphi_{i}\right)=800+80+2+378=1260$. Since the degree of $\operatorname{deg}\left(\prod_{i=1}^{14} C_{i}^{\prime} \varphi_{i}\right)$ is $36 \cdot 35=1260=\Delta_{36}^{2}$ we have $\operatorname{deg}\left(\prod b_{i}\right)=1$, since $\forall i, b_{i}$ is a positive power of a positive halftwist, we get $b_{i}=1 \forall i$. So we have $\Delta_{36}^{2}=\prod_{i=1}^{14} C_{i}^{\prime} \tilde{\varphi}_{i}$.

3.3.1. Invariance rules. The aim of this subsection is to prove that indeed $\Delta_{36}^{2}=$ $\prod_{i=1}^{14} C_{i}^{\prime} \varphi_{i}$. For this, we need to define a few definitions. We start by defining a Hurwitz move on $G \times \cdots \times G$ ( $G$ is a group) or on a set of factorizations.

Definition: Hurwitz moves:

Let $\vec{t}=\left(t_{1}, \ldots, t_{m}\right) \in G^{m}$. We say that $\vec{s}=\left(s_{1}, \ldots, s_{m}\right) \in G^{m}$ is obtained from $\vec{t}$ by the Hurwitz move $R_{k}$ (or $\vec{t}$ is obtained from $\vec{s}$ by the Hurwitz move $\left.R_{k}^{-1}\right)$ if

$$
s_{i}=t_{i} \quad \text { for } i \neq k, k+1, s_{k}=t_{k} t_{k+1} t_{k}^{-1}, s_{k+1}=t_{k} .
$$




\section{Definition: Hurwitz move on a factorization}

Let $G$ be a group $t \in G$. Let $t=t_{1} \cdot \ldots \cdot t_{m}=s_{1} \cdot \ldots \cdot s_{m}$ be two factorized expressions of $t$. We say that $s_{1} \cdot \ldots \cdot s_{m}$ is obtained from $t_{1} \cdot \ldots \cdot t_{m}$ by a Hurwitz move $R_{k}$ if $\left(s_{1}, \ldots, s_{m}\right)$ is obtained from $\left(t_{1}, \ldots, t_{m}\right)$ by a Hurwitz move $R_{k}$. Definition: Hurwitz equivalence of factorization

Two factorizations are Hurwitz equivalent if they are obtained from each other by a finite sequence of Hurwitz moves.

Definition: A factorized expression invariant under $h$

Let $t=t_{1} \cdot \ldots \cdot t_{m}$ be a factorized expression in a group $G$. We say that $t$ is invariant under $h \in G$ if $\left(t_{1}\right)_{h} \cdot \ldots \cdot\left(t_{m}\right)_{h}$ is a Hurwitz equivalent to $t_{1} \cdot \ldots \cdot t_{m}$.

We cite now two lemmas that we will need below.

Lemma 3.2. (see [5]) If a braid monodromy factorization of $\Delta_{36}^{2}=\prod \varphi\left(\Gamma_{i}\right)$ (where $\Gamma_{i}$ is a g-base: a free base of $\pi_{1}\left(\mathbb{C}^{1}-N, u\right)$ with certain properties; see [5] for definition) is invariant under $h$, then the equivalent factorization $\prod\left(\varphi\left(\Gamma_{i}\right)\right)_{h}=: \prod Z_{i}$ is also a braid monodromy factorization. That is, $\exists$ a g-base $\Gamma_{i}^{\prime}$ of $\pi_{1}\left(\mathbb{C}^{1}-N, u\right)$ s.t. $Z_{i}=\varphi\left(\Gamma_{i}^{\prime}\right)$.

Lemma 3.3 (Chakiri's Lemma). Let $t=t_{1} \cdot \ldots \cdot t_{m}$ be a factorized expression in a group $G$. Then $t_{1} \cdot \ldots \cdot t_{m}$ is invariant under $t^{k}, \forall k \in \mathbb{Z}$.

We now look at all the invariance relations that are related to any kind of point.

Lemma 3.4. $\forall i, \forall\left(m_{j}\right)_{1 \leq j \leq 18} \in \mathbb{Z}, C_{i}^{\prime}$ is invariant under $\varepsilon=\prod_{i=1}^{18} Z_{j j^{\prime}}^{m_{j}}$.

Lemma 3.5. $\forall i$, s.t. $v_{i}$ is a 3-point, $v_{i}=L_{\alpha} \cap L_{\beta}, \varphi_{i}$ is invariant under $\varepsilon$.

Proof: [8], Corollary 14.

Lemma 3.6. $\forall i$, s.t. $v_{i}$ is a 2-point or an extra branch point, $\varphi_{i}$ is invariant under $\varepsilon$.

Proof: the 2-points are $v_{1}, v_{12}$; consider $v_{1}$ (recall that $v_{12}$ does not contribute a factor to our factorization). We have to check if $Z_{1,1^{\prime}}$ is invariant under $Z_{j, j^{\prime}} \forall j$. For $j \neq 1, Z_{1,1^{\prime}}$ is invariant under $Z_{j, j^{\prime}}$ (since the paths corresponding 
to the braids are disjoint; thus the braids commute). For $j=1$ we have invariance by Chakiri's Lemma. For $v_{14}$, we apply the same procedure.

Corollary 3.6. $\varphi_{5}$ is invariant under $\left(Z_{1,1^{\prime}} Z_{12,12^{\prime}}\right)^{p_{1}}\left(Z_{2,2^{\prime}} Z_{11,11^{\prime}}\right)^{q_{1}}\left(Z_{3,3^{\prime}} Z_{6,6^{\prime}}\right)^{r_{1}}$, $\varphi_{6} \quad$ is invariant under $\left(Z_{4,4^{\prime}} Z_{14,14^{\prime}}\right)^{p_{2}}\left(Z_{5,5^{\prime}} Z_{13,13^{\prime}}\right)^{q_{2}}\left(Z_{6,6^{\prime}} Z_{8,8^{\prime}}\right)^{r_{2}}$, $\forall p_{i}, q_{i}, r_{i} \in \mathbb{Z}, i=1,2$.

Proof: This is the same as Lemma 15 in [8].

We shall prove now the invariance property for the 6-PT2.

Lemma 3.7. $\varphi_{7}=\prod_{\substack{i=8,9 \\ 15,17}} Z_{i, i^{\prime}}^{-1} Z_{7,7^{\prime}}^{-2} \Delta_{12}^{2}$.

Proof: Let

$$
\begin{gathered}
L=\left\{i, i^{\prime} \mid 1 \leq i \leq 18, i \neq 7,8,9,15,16,17\right\} \\
G=\left\{b \in B_{36}\left[D,\left\{i, i^{\prime} \mid i=1, \ldots, 18\right\}\right] \mid b\{L\}=\{L\}\right\} .
\end{gathered}
$$

Denote $\nu: G \rightarrow B_{12}\left[D,\left\{i, i^{\prime} \mid i=7,8,9,15,16,17\right\}\right]$ the forgetting homomorphism. Thus - $\nu\left(\Delta_{36}^{2}\right)=\Delta_{12}^{2}$; each factor in $C_{i}^{\prime}$ contains one of the indices in $L$, so $\nu\left(C_{i}^{\prime}\right)=1 \forall i$.

For $i=1,2,4,5,9,10,11$ all of the indices in $\tilde{\varphi}_{i}$ are in $L$, and so $\nu\left(\tilde{\varphi}_{i}\right)=1$ for $i=1,3,8,11,13 v_{i}$ is a 3 -point, when only one index of $\tilde{\varphi}_{i}$ is in $L$. So we have (by [8], lemma 2)

$$
\nu\left(\tilde{\varphi}_{3}\right)=Z_{7,7^{\prime}}, \nu\left(\tilde{\varphi}_{8}\right)=Z_{9,9^{\prime}}, \nu\left(\tilde{\varphi}_{11}\right)=Z_{15,15^{\prime}}, \nu\left(\tilde{\varphi}_{13}\right)=Z_{17,17^{\prime}}
$$

. For $i=12, v_{i}$ is a 2 -point that does not contribute a factor to the factorization. For $i=14, v_{i}$ is an extra branch point, so $\nu\left(\tilde{\varphi}_{14}\right)=Z_{7,7^{\prime}} . v_{6}$ is a 6-PT1. All of the factors outside $\hat{F}_{6,1}$ contain indices in L. So $\nu\left(\tilde{\varphi}_{6}\right)=\nu\left(\hat{F}_{6,1}\left(\hat{F}_{6,1}\right)_{\rho_{6}^{-1}}\right)=Z_{8,8^{\prime}}$. Thus $\Delta_{12}^{2}=\nu\left(\tilde{\varphi}_{7}\right) \prod_{\substack{i=8,9 \\ 15,17}} Z_{i, i^{\prime}} Z_{7,7^{\prime}}^{2}$, and so $h_{7}$ commutes with $\Delta_{12}^{2} \prod_{\substack{i=8,9 \\ 15,17}} Z_{i, i^{\prime}}^{-1} Z_{7,7^{\prime}}^{-2}$; Therefore, $\nu\left(\tilde{\varphi}_{7}\right)=\nu\left(\varphi_{7}\right)$; and since the indices in $L$ do not appear in $\varphi_{7}, \nu\left(\varphi_{7}\right)=$ $\varphi_{7}$. Thus,

$\varphi_{7}=\prod_{\substack{i=8,9 \\ 15,17}} Z_{i, i^{\prime}}^{-1} Z_{7,7^{\prime}}^{-2} \Delta_{12}^{2}$.

Corollary 3.7. $\varphi_{7}$ is invariant under $I_{1}(p)=\left(Z_{8,8^{\prime}} Z_{9,9^{\prime}}\right)^{p}\left(Z_{15,15^{\prime}} Z_{17,17^{\prime}} Z_{7,7^{\prime}}^{2}\right)^{p}$ $\forall p \in \mathbb{Z}$. 
Proof: We know that $\varphi_{7}=\prod_{\substack{i=8,9 \\ 15,17}} Z_{i, i^{\prime}}^{-1} Z_{7,7^{\prime}}^{-2} \Delta_{12}^{2}$. By Chakiri's Lemma, $\varphi_{7}$ is invariant under $\left(\prod_{\substack{i=8,9 \\ 15,17}} Z_{i, i^{\prime}}^{-1} Z_{7,7^{\prime}}^{-2} \Delta_{12}^{2}\right)^{-p}$. Since $\Delta_{12}^{2}$ is a central element, $\varphi_{7}$ is invariant under $\left(Z_{8,8^{\prime}} Z_{9,9^{\prime}}\right)^{p}\left(Z_{15,15^{\prime}} Z_{17,17^{\prime}} Z_{7,7^{\prime}}^{2}\right)^{p}$.

Denote - $\vartheta=Z_{15,16}^{-2} Z_{15,16^{\prime}}^{-2} Z_{16^{\prime}, 17}^{2} Z_{16,17}^{2}$.

Corollary 3.8. $\varphi_{7}$ is invariant under $I_{2}(p)=\rho^{p}=\left(Z_{7,7^{\prime}} Z_{16,16^{\prime}}\right)^{p}$

Proof: Looking at the factors outside $\left(\tilde{F}(\tilde{F})_{\rho^{-1}}\right)^{\vartheta}$, we can see, using the invariance rules 2 and $3([8])$, that outside $\left(\tilde{F}(\tilde{F})_{\rho^{-1}}\right)^{\vartheta}$, the factorization is indeed invariant under $Z_{7,7^{\prime}}$ and $Z_{16,16^{\prime}}$, and by the invariance remark (v) [8], is invariant under $\rho$. So it is enough to check that $\left(\tilde{F}(\tilde{F})_{\rho^{-1}}\right)^{\vartheta}$ is invariant under $\rho$. But this is proven exactly in the same way as in Lemma 15, case 2.2,[8] (since in our case the point $v_{7}$ and in the standard case of the 6 -point at [8], the regeneration of the 4 -point are the same).

Corollary 3.9. $\varphi_{7}$ is invariant under $I_{3}(p)=\left(Z_{8,8^{\prime}} Z_{9,9^{\prime}}\right)^{p}$.

Proof: We use the invariance rules ([8]) when passing on all the factors of $\varphi_{7}$. We use invariance rules 2 and 3 when passing on the factors outside $\left(\tilde{F}(\tilde{F})_{\rho^{-1}}\right)^{\vartheta}$ (for example, by invariance rule number $3,\left(Z_{8,8^{\prime}, 15}^{(3)}\right)_{16^{\prime}, 17}^{2} Z_{16,17}^{2}$ is invariant under $Z_{8,8^{\prime}}$; by invariance rule number $2, Z_{9^{\prime}, 15}^{2} Z_{9,15}^{2}$ is invariant under $\left.Z_{9,9^{\prime}}\right)$. When passing on the factors of $\left(\tilde{F}(\tilde{F})_{\rho^{-1}}\right)^{\vartheta}$, we use invariance rule 3 (for factors of the form $\left.Z_{\ldots}^{(3)}\right)$ and invariance rule 1 (for the factors $Z_{8^{\prime}, 9} Z_{8,9^{\prime}}$ and $\left.\left(Z_{8^{\prime}, 9} Z_{8,9^{\prime}}\right)_{\rho^{-1}}\right)$.

Corollary 3.10. $\varphi_{7}$ is invariant under $\left(Z_{8,8^{\prime}} Z_{9,9^{\prime}}\right)^{q}\left(Z_{15,15^{\prime}} Z_{17,17^{\prime}} Z_{7,7^{\prime}}^{2}\right)^{p}$ $\left(Z_{7,7^{\prime}} Z_{16,16^{\prime}}\right)^{r}, \forall p, q, r \in \mathbb{Z}$.

Proof: By invariant remark (v) ([8]), $\varphi_{7}$ is invariant under $I_{3}(q-p) \cdot I_{1}(p) \cdot I_{2}(r)$, which is the desired expression. 
The Main Result: As a consequence of the invariance rules, we can apply

them as in [8] and get that $\varepsilon(36):=\prod_{i=1}^{14} C_{i}^{\prime} \varphi_{i}$ is also a braid monodromy factorization.

Note, that although that the invariance rules for $v_{7}$ are different from the invariance rules of the standard 6-point, what matters, as can be seen in [8], Section 4 , is that the invariance rule regarding the horizontal lines in the 6-point (the two lines that are regenerated last) remains the same both in $v_{7}$ and in the standard 6-point.

\section{REFERENCES}

[1] D. Auroux, S. K. Donaldson, L. Katzarkov, M. Yotov, Fundamental groups of complements of plane curves and symplectic invariants, Topology 43, , (2004) 1285-1318.

[2] V. S. Kulikov and M. Teicher, Braid monodromy factorizations and diffeomorphism types, Izv. Ross. Akad. Nauk Ser. Mat. 64(2), (2000), 89-120 , [Russian]; English transl., Izvestiya Math. 64(2), (2000), 311-341.

[3] V. Kharlamov and Vik. S. Kulikov, Diffeomorphisms, isotopies, and braid modromy factorizations of plane cuspidal curves, C.R. Acad. Sci., Série I, Paris, (2001) t.333, 5.

[4] B. Moishezon, A. Robb and M. Teicher, On Galois covers of Hirzebruch surfaces, Math. Ann. 305, (1996), 493-539 .

[5] B. Moishezon and M. Teicher,Braid group technique in complex geometry, I, Line arrangements in $\mathbb{C P}^{2}$, Contemp. Math. 78, (1988), 425-555.

[6] B. Moishezon and M. Teicher, Braid group technique in complex geometry, II, From arrangements of lines and conics to cuspidal curves, Algebraic Geometry, Lecture Notes in Math., (1990), vol. 1479.

[7] B. Moishezon and M. Teicher, Braid group techniques in complex geometry III: Projective degeneration of V3, Contemp. Math. 162, (1993), 313-332.

[8] B. Moishezon and M. Teicher, Braid group techniques in complex geometry IV: Braid monodromy of the branch curve $S_{3}$ of $V_{3} \rightarrow \mathbb{C P}^{2}$ and application to $\pi_{1}:\left(\mathbb{C P}^{2}-S_{3}, *\right)$, Contemp. Math. 162, (1993), 332-358.

[9] B. Moishezon and M. Teicher, Braid group techniques in complex geometry, V: The fundamental group of complements of a branch curve of Veronese generic projection, Communications in Analysis and Geometry 4, (1996), no. 1, 1-120.

[10] A. Robb, The topology of branch curves of complete intersections, Doctoral Thesis, Columbia University, (1994).

[11] A. Robb, On branch curves of Algebraic Surfaces, Stud. Adv. Math. 5, (1997), 193-221.

[12] M. Teicher, New invariants for surfaces, Contemp. Math. 231, (1999), 271-281.

[13] E.R. Van Kampen, On the fundamental group of an algebraic curve, Amer. J. Math. 55 (1933), 255-260. 
Michael Friedman

Department of Mathematics, Bar-Ilan University

52900 Ramat Gan, Israel

E-mail: fridmam@mail.biu.ac.il

Mina Teicher

Department of Mathematics, Bar-Ilan University 52900 Ramat Gan, Israel

E-mail: teicher@macs.biu.ac.il 